\title{
The Shapes of Flux Domains in the Intermediate State of Type-I Superconductors
}

\author{
Alan T. Dorsey* \\ Department of Physics, University of Florida, Gainesville, FL 32611 \\ Raymond E. Goldstein** \\ Department of Physics and Program in Applied Mathematics \\ University of Arizona, Tucson, AZ 85721
}

(March 25, 2018)

\begin{abstract}
In the intermediate state of a thin type-I superconductor magnetic flux penetrates in a disordered set of highly branched and fingered macroscopic domains. To understand these shapes, we study in detail a recently proposed "current-loop" (CL) model [R.E. Goldstein, D.P. Jackson, A.T. Dorsey, Phys. Rev. Lett. 76, 3818 (1996)] that models the intermediate state as a collection of tense current ribbons flowing along the superconducting-normal interfaces and subject to the constraint of global flux conservation. The validity of this model is tested through a detailed reanalysis of Landau's original conformal mapping treatment of the laminar state, in which the superconductor-normal interfaces are flared within the slab, and of a closely-related straight-lamina model. A simplified dynamical model is described that elucidates the nature of possible shape instabilities of flux stripes and stripe arrays, and numerical studies of the highly nonlinear regime of those instabilities demonstrate patterns like those seen experimentally. Of particular interest is the buckling instability commonly seen in the intermediate state. The free-boundary approach further allows for a calculation of the elastic properties of the laminar state, which closely resembles that of smectic liquid crystals. We suggest several new experiments to explore of flux domain shape instabilities, including an Eckhaus instability induced by changing the out-of-plane magnetic field, and an analog of the Helfrich-Hurault instability of smectics induced by an in-plane field.
\end{abstract}

\section{INTRODUCTION}

A longstanding problem in macroscopic superconductivity is that of understanding the complex patterns of flux penetration observed in the intermediate state of a type-I superconductor. This state is observed when a thin superconducting slab is placed in a perpendicular magnetic field. Unlike type-II superconductors, where the field penetration is in the form of tubes each with a quantum of magnetic flux, type-I systems are observed to form intricately branched and fingered macroscopic flux domains [1 3]. Thus, instead of establishing a Meissner phase, in which the magnetic induction $\mathbf{B}=0$ uniformly, the demagnetizing effects of the large aspect ratio force the sample to break up into regions, some of which are uniformly superconducting (with $\mathbf{B}=\mathbf{0}$ inside) and others that are normal $(\mathbf{B} \neq 0)$. Figure 1 shows a typical example of these patterns [3. The superconducting regions appear black, having been decorated with a powder (niobium) that is itself superconducting at the sample temperature and thus migrates to the regions of low magnetic field. Other imaging techniques include Hall probes [4] and magneto-optics [5]. All reveal similar structures.

The sample in Fig. 1 is at an applied magnetic field $H_{a}$ that is very close to the critical field $H_{c}$ at which the sample would be completely normal, so the minority phase is superconducting. Similar patterns are observed when $H_{a} / H_{c}$ is very small, but now the minority phase

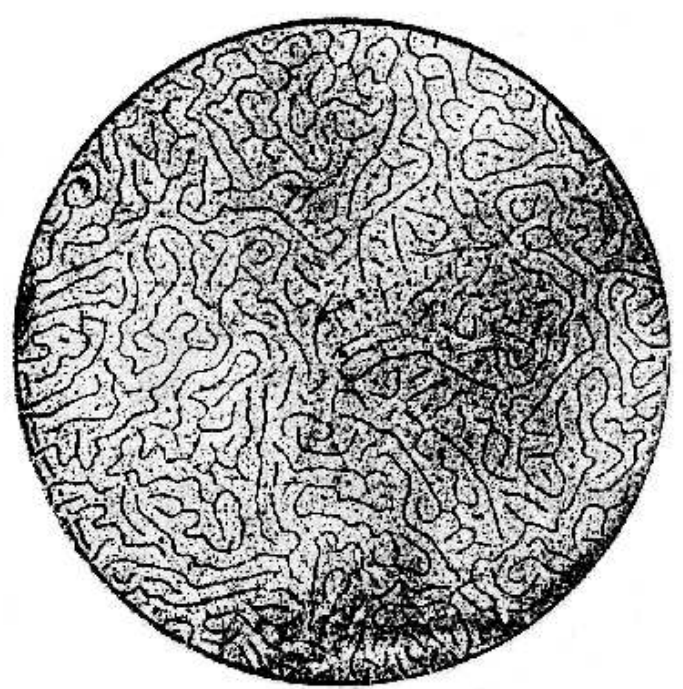

FIG. 1. The intermediate state of a thin slab of indium, in which the superconducting regions (black) are decorated with niobium (black). The applied field $H_{a}$ is close to the critical field $H_{c}\left(h=H_{a} / H_{c}=0.931\right)$. Adapted from Haenssler and Rinderer [3]. 
is normal; the sample consists of fingered and branched flux domains in a matrix of superconductor. These domains have a characteristic field-dependent finger width, and the branched domains have three-fold vertices.

For many years it has been known [6] that more ordered flux domain states may be observed in the presence of a small in-plane component of the applied magnetic field. It is also known that the domain morphology is not a thermodynamic state function; it depends on the path in field-temperature space through which the sample has been brought to a given point [3]. Thus, for instance, cooling in zero field below the transition temperature and then applying the field tends to produce patterns in which normal domains are embedded in a matrix of superconductor, whereas when the same point in $T-H$ space is reached by cooling below $T_{c}$ in a fixed field the normal domains connect to the sample edges [1]. These observations suggest that the patterns are not true ground states of the system - the sample is not in true thermodynamic equilibrium.

Despite the ubiquity of these patterns, there has until recently been no theoretical explanation for their form. The earliest attempt, prior to the detailed experiments described above, was by Landau [7], who considered the laminar state: a periodic array of alternating superconducting and normal domains (Fig. 2). Exploiting the translational invariance of the pattern in the direction parallel to the stripes, the cross-sectional shape of the domain walls and the associated bending of the magnetic field lines become purely two-dimensional problems amenable to conformal mapping techniques. The primary result of this calculation is a determination of the laminar state free energy as a function of lamina spacing, applied field, superconductor-normal surface energy, and slab thickness.

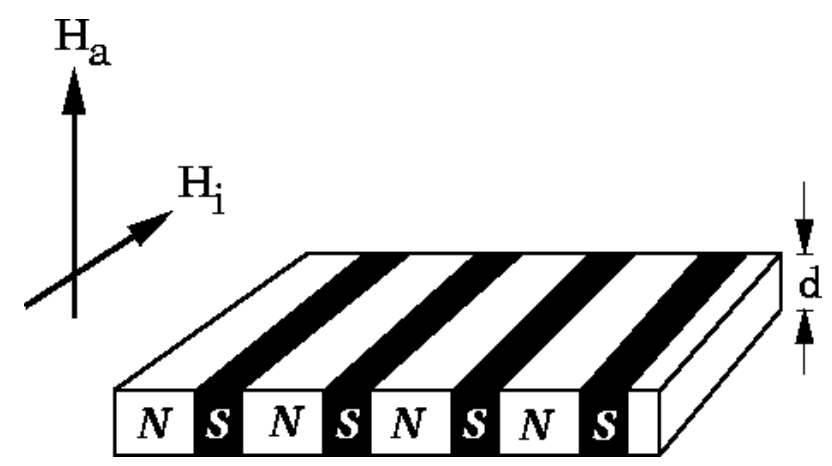

FIG. 2. The laminar state in a thin slab. The applied field $H_{a}$ is normal to the slab. An ordered laminar structure is obtained with an additional in-plane component $H_{i}$.

The free energy so obtained depends on two parameters: the area fraction occupied by the normal state and the repeat distance of the structure. For thick slabs, one finds to a good approximation that the equilibrium area fraction is set by the reduced field $h=H_{a} / H_{c}$.
Deep within the slab the domain walls are aligned with the field, but they flare along with the magnetic field lines near the surface. The equilibrium field-dependent width has been found to be in good agreement with subsequent experiments [1-3]. Stability calculations about this state are, however, precluded by the reliance on conformal mapping techniques, and no systematic calculations of this type have been performed. Subsequently, and in light of experiments showing nearly circular flux domains, there have been several calculations of the energies of periodic arrays of simple geometric structures [8]. These studies generally find that the free energies of these periodic structures are very close to each other, so there is little thermodynamic driving force preferring one over the other. None of these theories offers an explanation for the appearance of branched flux domains.

The dual features of (i) disordered domain shapes and (ii) path dependence to the patterns makes it clear that an understanding of the intermediate state requires a theoretical approach that (a) treats the superconductornormal (SN) interfaces as a free-boundary problem, and (b) addresses the dynamics of those interfaces. That one should consider an interfacial representation at all, rather than a more microscopic approach based on equations of motion for the superconducting order parameter and the vector potential, is made clear by the strong separation of scales that exists between the domain size (typically fractions of a millimeter) and the width of the SN interface. For a strongly type-I superconductor the width is set by the coherence length $\xi$, which is on the order of $0.2 \mu \mathrm{m}$.

Several recent studies [9, 10] have emphasized strong connections between the motion of SN interfaces in the presence of a magnetic field and the dynamics of solidliquid interfaces in the process of solidification. The key to this relationship is the fact that the magnetic field in the normal state obeys a diffusion equation 111, analogous to the diffusion of latent heat in the solidification problem. These suggest that a diffusional instability like the Mullins-Sekerka instability [12] should occur in the process of flux invasion. Numerical studies of the timedependent Ginzburg-Landau model confirmed these instabilities, which can lead to highly ramified domain patterns.

It was also found by asymptotic methods [13] that in the limit of a sharp interface it is possible to integrate out the magnetic field contributions and arrive at a nonlocal free-boundary theory for the SN interface alone. The nonlocality is both temporal and spatial, the latter taking the form of a Biot-Savart interaction between distant segments of the interface. The appearance of this type of coupling reminds us that the supercurrents that provide the screening of the applied magnetic field flow along the SN interfaces. Many years ago Pearl [14, and later Fetter and Hohenberg [15] showed that the interactions between currents flowing in thin slabs are long-ranged. 
Thus, vortices in a thin film interact with a potential whose long-range form is unscreened, $V(r) \sim 1 / r$, while at short distances $V(r) \sim \ln (\Lambda / r)$, where $\Lambda$ is a cutoff. Screening is unimportant at long distances because the interaction energy is dominated by the electromagnetic fields (in vacuo) above and below the slab.

Based on all of these observations, we recently proposed [16] a "current-loop" (CL) model as an approximate description of the intermediate state. The model describes the patterns as a collection of tense ribbons of current, interacting with the long-range Biot-Savart interaction of currents in free space, and subject to a constraint of global flux conservation. It is based on several simplifying assumptions. First, the domain walls are taken to be vertical, rather than flared as in Landau's more precise calculation. Second, only the supercurrents in those walls are considered - surface supercurrents on the top and bottom of the sample are ignored. These approximations allow for an averaging process over the slab thickness that maps the simplified model onto one of self-interacting contours in the plane. Such freeboundary approaches are well-known for hydrodynamic problems such as Saffman-Taylor fingering [17, as well as in systems governed by partial differential equations of the reaction-diffusion type. In the latter case, the dynamics may be reduced to that of an interface when the scale of the patterns is large compared to the width of the front connecting two locally stable states.

While several important phenomena are precluded from study in this model (such as domain fission), it has the virtue of providing a simple picture of the mechanism of shape instabilities in this system, and may form a useful starting point for a more precise treatment 18]. It also is strikingly similar to models for the energetics and dynamics of interfacial pattern formation in a variety of other systems that display "labyrinthine" patterns from a competition between interfacial tension and long-range electromagnetic interactions [19]. These include fingering instabilities of magnetic fluids in Hele-Shaw flow 20 24, Langmuir monolayers at the air-water interface [25] 27$]$ and thin magnetic films 28. It is also equivalent to a reaction-diffusion system 29] 32 in which chemical fronts between two locally stable states may form space-filling disordered labyrinths similar to those seen in experiments on chemical pattern formation in a gel reactor [33, 34 .

In Section II we set the stage for a macroscopic model of the intermediate state by reviewing both the sharp-interface limit of the time-dependent GinzburgLandau model for nonequilibrium superconductivity and the derivation of long-range interactions between currents in the slab geometry. The conformal mapping solution to Landau's model of the intermediate state and the simpler straight-lamina model are derived in Section III, in which careful attention is paid to the consequences of those long-range interactions. The structure of the free energy as a function of stripe periodicity and width, surface tension and applied field in the two models is shown to be essentially equivalent, as are their predictions for the equilibrium stripe width as a function of field. The current-loop model is described and applied to the energetics of the laminar state in Section IV. Instabilities of single flux stripes and the elastic properties of the laminar state are found in Section V. The concluding Section VI summarizes the new experimental predictions that arise from the correspondence between the intermediate state and others such as Langmuir monolayers and smectic liquid crystals. An appendix gathers together calculational details of the stability analyses and elasticity calculations.

\section{PROPERTIES OF THE MACROSCOPIC MODEL}

\section{A. The sharp-interface limit}

Here we review the results of asymptotic methods applied to the time-dependent Ginzburg-Landau (TDGL) model of superconductivity in the limit of sharp interfaces. The microscopic parameters that enter the TDGL include the charge $e^{*}$ and mass $m$ of a Cooper pair, a dimensionless order parameter relaxation time $\gamma$, and the conductivity $\sigma$ of the normal phase. The coupled equations of motion for the order parameter $\psi$ and the scalar and magnetic vector potentials, $\phi$ and $\mathbf{A}$, are

$$
\begin{aligned}
\hbar \gamma\left(\partial_{t}+\frac{i e^{*}}{\hbar} \phi\right) \psi= & \frac{\hbar^{2}}{2 m}\left(\boldsymbol{\nabla}-\frac{i e^{*}}{\hbar} \mathbf{A}\right)^{2} \psi \\
& +a \psi-b|\psi|^{2} \psi, \\
\boldsymbol{\nabla} \times \boldsymbol{\nabla} \times \mathbf{A}= & 4 \pi\left(\mathbf{J}_{n}+\mathbf{J}_{s}\right) .
\end{aligned}
$$

where $\mathbf{J}_{n}$ and $\mathbf{J}_{s}$ are the normal and supercurrents,

$$
\begin{aligned}
& \mathbf{J}_{n}=\sigma\left(-\nabla \phi-\partial_{t} \mathbf{A}\right) \\
& \mathbf{J}_{s}=\frac{\hbar e^{*}}{2 m i}\left(\psi^{*} \boldsymbol{\nabla} \psi-\psi \boldsymbol{\nabla} \psi^{*}\right)-\frac{e^{* 2}}{m}|\psi|^{2} \mathbf{A} .
\end{aligned}
$$

The bifurcation parameter $a$ contains the important temperature dependence, $a=a_{0}\left(1-T / T_{c}\right)$ and controls the correlation length $\xi=\hbar /(2 m|a|)^{1 / 2}$ and penetration depth $\lambda=\left[m b / 4 \pi e^{* 2}|a|\right]^{1 / 2}$. Finally, the GinzburgLandau parameter $\kappa=\lambda / \xi$.

When $\xi$ is small the superconductor-normal interface will be sharp, and a natural measure of smallness is provided by the inverse distance from the critical point. Setting $a=\tilde{a} / \epsilon^{2}$ and rescaling all distances and fields with appropriate powers of $\epsilon$, one finds that both $\lambda$ and $\xi$ scale identically. The sharp-interface limit is then achieved by $\xi, \lambda \rightarrow 0$ with $\kappa$ fixed. It is thus possible in this limit to continue to distinguish between type-I $(\kappa<1 / \sqrt{2})$ and type-II $(\kappa>1 / \sqrt{2})$ systems. 
Existing derivations of interface equations of motion in this context presume translational invariance in the direction along which the magnetic field is applied, and hence describe "bulk" superconductors [13. The results may be summarized as follows. Far away from the interface (the "outer" solution in the sense of matched asymptotics), the magnetic field in the normal region obeys the diffusion equation

$$
\mathbf{h}_{t}=D \nabla^{2} \mathbf{h}
$$

with diffusion constant $D=1 / 4 \pi \sigma$. The boundary condition on the magnitude $h_{i}$ of $\mathbf{h}$ on the interface $\mathcal{C}$ is

$$
\left.h\right|_{\mathcal{C}}=H_{c}\left[1-\frac{4 \pi}{H_{c}^{2}}\left(\sigma_{S N} \mathcal{K}+\Gamma^{-1} v_{n}\right)\right] \text {. }
$$

where $\sigma_{S N}$ is the interfacial tension (with dimensions of energy/area), $\mathcal{K}$ is the interface curvature, $\Gamma$ is a known kinetic coefficient, and $v_{n}=\hat{\mathbf{n}} \cdot \mathbf{r}_{t}$ is the normal component of the interface velocity.

The equation of motion is an expression for $v_{n}$ in terms of the function $\mathbf{r}(s, t)$, and it follows from a solution to the diffusion equation (2.4) given the boundary conditions (2.5). As with all such problems, this involves a convolution over previous times and all space. But in the limit $D \rightarrow \infty$, or vanishing normal state conductivity, the temporal nonlocality disappears. This yields the equation of motion

$$
\begin{aligned}
\Gamma^{-1} \hat{\mathbf{n}} \cdot \mathbf{r}_{t}(s, t)= & \frac{H_{c}^{2}-H_{a}^{2}}{8 \pi}+\sigma_{S N} \mathcal{K} \\
& -\frac{H_{c}^{2}}{8 \pi^{2}} \oint d s^{\prime} \frac{\mathbf{R} \times \hat{\mathbf{t}}\left(s^{\prime}\right)}{R^{2}},
\end{aligned}
$$

with $\mathbf{R}=\mathbf{r}(s)-\mathbf{r}\left(s^{\prime}\right)$. The dynamics (2.6) has the variational form

$$
\mathbf{r}_{t}=-\Gamma \frac{1}{\sqrt{g}} \frac{\delta \mathcal{H}_{\mathrm{eff}}}{\delta \mathbf{r}}
$$

where $g$ is the interface metric and the effective interface Hamiltonian for a single domain is

$$
\begin{aligned}
\mathcal{H}_{\mathrm{eff}}[\mathbf{r}]= & -\frac{H_{c}^{2}-H_{a}^{2}}{8 \pi} A+\sigma_{S N} L \\
& -\frac{H_{c}^{2}}{8 \pi} \oint d s \oint d s^{\prime} \hat{\mathbf{t}}(s) \cdot \hat{\mathbf{t}}\left(s^{\prime}\right) \mathcal{G}(R),
\end{aligned}
$$

with $\mathcal{G}(R)=-(1 / 2 \pi) \ln R$ is the Green's function of the Laplacian in two dimensions, and $A$ and $L$ the area and perimeter of the domain. As anticipated in the introduction, we see that the energy associated with the distortion of the magnetic field lines threading a domain is represented by the self-induction of the boundary. The other contributions are simply the line energy $\sigma_{S N} L$ and an area term associated with the magnetic pressure and condensation energy.
The limits of a sharp interface and zero normal state conductivity that produce a temporally local but spatially nonlocal contour dynamics have parallels in a simpler reaction-diffusion system of recent interest 229 32. This is the FitzHugh-Nagumo model [35] of the coupled dynamics of an activator $u$ and inhibitor $v$, considered in two spatial dimensions,

$$
\begin{aligned}
u_{t} & =\bar{D} \nabla^{2} u-F^{\prime}(u)-\rho(v-u) \\
\epsilon v_{t} & =\nabla^{2} v-v+u
\end{aligned}
$$

These partial differential equations are written in a rescaled form in which the activator diffusion constant $\bar{D}$ is normalized to that of the inhibitor, while $\epsilon$ is a ratio of their characteristic times. The function $F(u)$, whose derivative $F^{\prime}(u)$ appears above, is a double-well potential that describes the auto-catalytic behavior (and bistability) of the activator. When $\epsilon \neq 0$, the coupled dynamics is not a gradient flow in any standard form.

It is clear that the two systems (2.2) and (2.9) share many features. In each case there is an order parameter field ( $\psi$ or $u$ ) with an underlying bifurcation (the former continuous, the latter of first order), which is coupled to a diffusing field (unscreened or screened). In the case of the superconductor, the second field could be integrated out of the problem in exchange for an instantaneous nonlocal coupling of the field $\psi$. An identical feature appears in the reaction-diffusion problem when the parameter $\epsilon$ is small, for then the inhibitor relaxes on times short compared to that of the activator and will be slaved to $u$. Setting $\epsilon v_{t}=0$, the field $v$ may then be expressed as $v=\int \mathcal{G} u$, where $\mathcal{G}$ is the appropriate Green's function for the modified Helmholtz operator. This produces the nonlocal dynamics for $u$ that $i s$ variational, $u_{t}=-\delta \mathcal{E} / \delta u$, with

$$
\begin{aligned}
\mathcal{E}[u]= & \int d^{2} x\left\{\frac{1}{2} \bar{D}|\nabla u|^{2}+F(u)-\frac{1}{2} \rho^{2}\right\} \\
& +\frac{1}{2} \rho \int d^{2} x \int d^{2} x^{\prime} u(\mathbf{x}) \mathcal{G}\left(\left|\mathbf{x}-\mathbf{x}^{\prime}\right|\right) u\left(\mathbf{x}^{\prime}\right) .
\end{aligned}
$$

Now taking the limit $\bar{D} \rightarrow 0$ we may reduce (2.10) to a functional of the contours bounding regions in which the activator takes on values corresponding to different minima of $F$. That new functional (of the position vectors $\mathbf{r}_{i}(s)$ for each domain boundary, parameterized by arclength $s$ ) is 29, 30 ]

$$
\begin{aligned}
\Delta \mathcal{E}\left[\left\{\mathbf{r}_{i}\right\}\right]= & \bar{\gamma} \sum_{i} L_{i}+\Delta F \sum_{i} A_{i} \\
& -\frac{\rho}{2} \sum_{i, j} \oint d s \oint d s^{\prime} \hat{\mathbf{t}}_{i} \cdot \hat{\mathbf{t}}_{j} \mathcal{G}\left(\left|\mathbf{r}_{i}-\mathbf{r}_{j}\right|\right)
\end{aligned}
$$

with $\bar{\gamma}$ the line tension, and where $L_{i}$ and $A_{i}$ the perimeter and enclosed area of each domain. The nonlocal term is again in the form of the self- and mutual-induction of (here fictitious) current loops encircling each of the domains. 


\section{B. Long-range forces}

As further motivation for the CL model, we recall Pearl's derivation 114] of the long-range (unscreened) potential between point vortices in a thin film. The starting point is the solution for the supercurrent surrounding a single vortex located at the origin. In the slab geometry, the order parameter has the simple form

$$
\psi(\mathbf{r})=\psi_{0} f(r) e^{i \theta} \quad(0 \leq z \leq d)
$$

where $\psi_{0}$ is the far-field value of $\psi$, which vanishes for $z<0$ and $z>d$. The function $f(r)$ describes the vortex core structure, and we take $f=1$ on the (large) scales of interest. The second of the Ginzburg-Landau pair of equations (2.2) is then

$$
\boldsymbol{\nabla} \times \boldsymbol{\nabla} \times \mathbf{A}=4 \pi \mathbf{J}_{s}=\frac{1}{\lambda^{2}}\left[\frac{\psi_{0}}{2 \pi r} \hat{\theta}-\mathbf{A}\right] .
$$

If the film is sufficiently thin, then we may average over the film thickness $d$ to obtain

$$
\boldsymbol{\nabla} \times \boldsymbol{\nabla} \times \mathbf{A}=\frac{d}{\lambda^{2}}\left[\frac{\psi_{0}}{2 \pi r} \hat{\theta}-\mathbf{A}\right] \delta(z) .
$$

In this form, it is readily apparent from the appearance of the term $-\left(d \mathbf{A} / \lambda^{2}\right) \delta(z)$ on the right-hand-side that the screening is confined solely to the slab.

The solution to this can be obtained through the use of Fourier-Hankel transforms and yields the supercurrent

$$
\mathbf{J}_{s}(r)=-\frac{\phi_{0}}{8 \pi \Lambda^{2}}\left[H_{1}(r / \Lambda)-Y_{1}(r / \Lambda)-\frac{2}{\pi}\right] \hat{\theta}
$$

where $H_{1}$ and $Y_{1}$ are Hankel functions, and the effective penetration depth for the thin film is

$$
\Lambda=\frac{2 \lambda^{2}}{d} \text {. }
$$

The interaction potential of a second point vortex placed a distance $r$ from the first is obtained by multiplying (2.15) by $\phi_{0}$ and integrating, with the result

$$
V(r)=\frac{\phi_{0}^{2}}{8 \pi \Lambda}\left[H_{0}(r / \Lambda)-Y_{0}(r / \Lambda)\right] .
$$

At long distances $r / \Lambda \gg 1$, this is an unscreened potential,

$$
V(r) \simeq \frac{\phi_{0}^{2}}{4 \pi^{2} r},
$$

whereas as $r \rightarrow 0$ the familiar logarithmic interaction between vortices appears,

$$
V(r) \simeq \frac{\phi_{0}^{2}}{4 \pi^{2} \Lambda} \log \left(\frac{e^{C} r}{2 \Lambda}\right),
$$

with $C$ being Euler's constant. We anticipate therefore that the interactions between Meissner currents in a thin type-I slab should have a similar long-range character, and now proceed to a detailed calculation.

\section{THE LAMINAR STATE}

\section{A. Landau's free-boundary solution}

In this first part of our discussion, we calculate the shape and optimal spacing for an assumed laminar geometry for the intermediate state. While our work reproduces Landau's original calculation [7], our method is quite different, and we can provide an explicit expression for the function $f(h)$ (see below). We are including this material in our discussion since (1) Landau's derivation is, to our taste, a bit obscure, so we hope that the present derivation will clarify the techniques and inspire further work on extending the calculations to more complicated geometries; and (2) the "exact" result derived here, for flared normal lamina, can be compared against the results of the current loop model discussed below, allowing us to "calibrate" the current loop model.

We consider the simplest semi-infinite geometry here, which we have illustrated in Fig. 3. The surface of the material is in the $x-y$ plane, occupying the region $z<0$. The field is applied perpendicular to the sample, so that $\mathbf{H}_{a}=H_{a} \hat{z}$. We will assume that there is an array of normal-superconducting laminae periodic in the $x$-direction, the laminae being straight in the $y$-direction. The normal and superconducting laminae have asymptotic widths $a_{n}$ and $a_{s}$, respectively, so that the period of the entire structure is $a=a_{s}+a_{n}$.

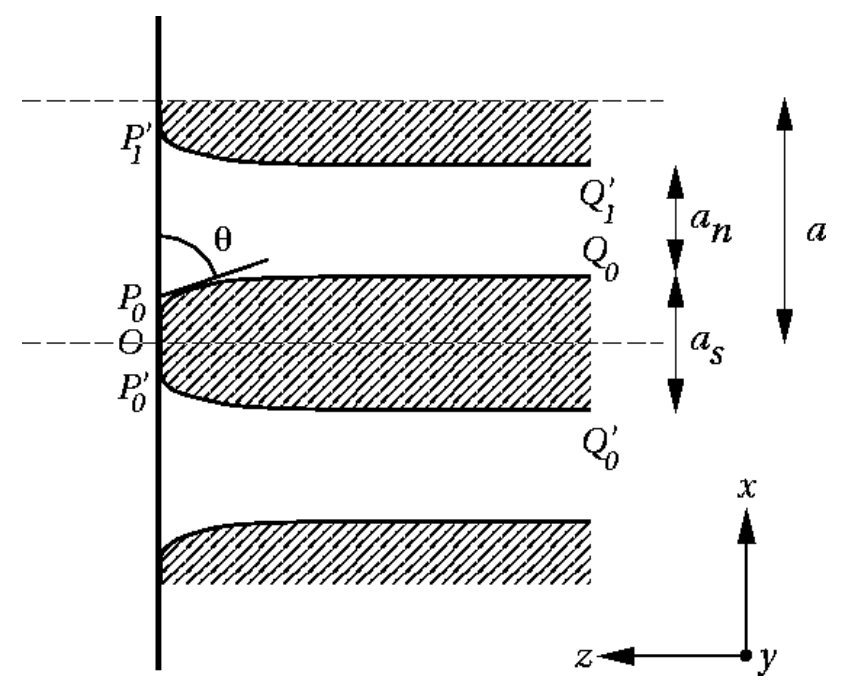

FIG. 3. Laminae in the intermediate state. Superconducting regions are shaded. Reference points $P_{n}, Q_{n}, P_{n}^{\prime}$, etc. are discussed in text.

We start by noting that the typical lamina spacing is large compared to the superconducting penetration depth and the coherence length, so that the interfaces separating the normal and superconducting phase are sharp. Then, following Landau, we can work with the macroscopic Maxwell equations. In the normal regions 
we have $\nabla \times \mathbf{B}=0, \nabla \cdot \mathbf{B}=0$, which can be solved either by introducing the scalar potential $\phi$ through $\mathbf{B}=-\nabla \phi$, so that $\nabla^{2} \phi=0$, or the vector potential $\mathbf{A}$ through $\mathbf{B}=\nabla \times \mathbf{A}$, which in the gauge $\nabla \cdot \mathbf{A}=0$ satisfies $\nabla^{2} \mathbf{A}=0$. We thus need to solve Laplace's equation in the $x-z$ plane. This immediately suggests the use of complex variable methods; indeed, for the laminar structure the only nonzero component of the vector potential is $A_{y}$, so we may introduce the complex potential

$$
w=\phi+i \psi=\phi+i A_{y}
$$

so that the complex magnetic field is

$$
B=B_{x}-i B_{z}=-\frac{d w}{d \zeta}, \quad \zeta=x+i z .
$$

The boundary conditions are the following. First, as $z \rightarrow$ $\infty$ the field becomes the uniform applied field $H_{a} \hat{z}$, so that

$$
w \sim i H_{a} \zeta, \quad z \rightarrow \infty
$$

Since the magnetic field vanishes in the superconducting regions, continuity of its normal component implies that the field is purely tangential both along the normalsuperconducting interface $P_{n} Q_{n}$ and along the segment which we refer to as the "nose" $\left(O P_{n}\right)$ (Fig. 3). In the first case, the assumption of local thermodynamic equilibrium at this interface implies that the magnitude $H_{n}$ of the tangential component is a constant (as yet unknown), so that the vector potential $A_{y}$ is also constant along any one interface. Along the nose the magnetic field is parallel to the surface $\left(\mathbf{H}=H_{x} \hat{x}\right)$ but with a magnitude that is no longer constant. The field $H_{n}$ along with the periods $a_{n}$ and $a_{s}$ are determined a posteriori by minimizing the energy. However, these parameters are not completely independent, since by flux conservation we must have $H_{a} a=H_{n} a_{n}$. Therefore for fixed external field $H_{a}$ the energy will be determined by $a$ and $a_{n}$.

\section{Exact determination of the lamina shape}

The position of the interface separating the normal and superconducting regions is not known a priori, and must be discovered in the process of solving the problem. Although this sounds like a formidable task, it is made easier by recognizing that our magnetostatics problem is formally equivalent to the flow of an ideal incompressible fluid around an array of plates, the plates being the noses of the laminae. The field lines would be the streamlines of the fluid; the normal-superconducting interfaces correspond to fluid streamlines which have separated from the flow behind the plate ("free-streamlines"). This correspondence is outlined in more detail in Table II. The shape of the free-streamlines can be determined by using the hodograph method [36,37]. The idea is that while the field lines in the $\zeta$-plane may be complicated, the representation of the field configuration in the $w$ and $H$ planes is quite simple; a suitable conformal transformation which maps the $w$ plane onto the $H$ plane would then lead to a relation between $w$ and $d w / d \zeta$, the solution of which will determine the shape of the interface. Consider first the $w$-plane. Since the magnetic field is tangent to both the nose segment and to the normalsuperconducting interface, the magnitude of the vector potential is constant on these segments, as well as on the centerline shown dashed in Fig. 3. Far from the sample (at a fixed $z \rightarrow \infty$ ), we know that $A_{y} \sim H_{a} x$; therefore, for laminae separated by a distance $a$, the vector potential on the interface of the $n-$ th lamina is $A_{y}=H_{a} n a$. The potential $\phi$ behaves as $-z H_{a}$ as $z \rightarrow \infty$, hence the lamina correspond to the positive half of the $\phi$ axis. The field configuration in the $w$-plane is shown in Fig. 1(a). Next we consider the $H$-plane; this is simpler if we introduce the normalized magnetic field

$$
\eta=\frac{H}{H_{n}}=-\frac{1}{H_{n}} \frac{d w}{d \zeta} .
$$

a)
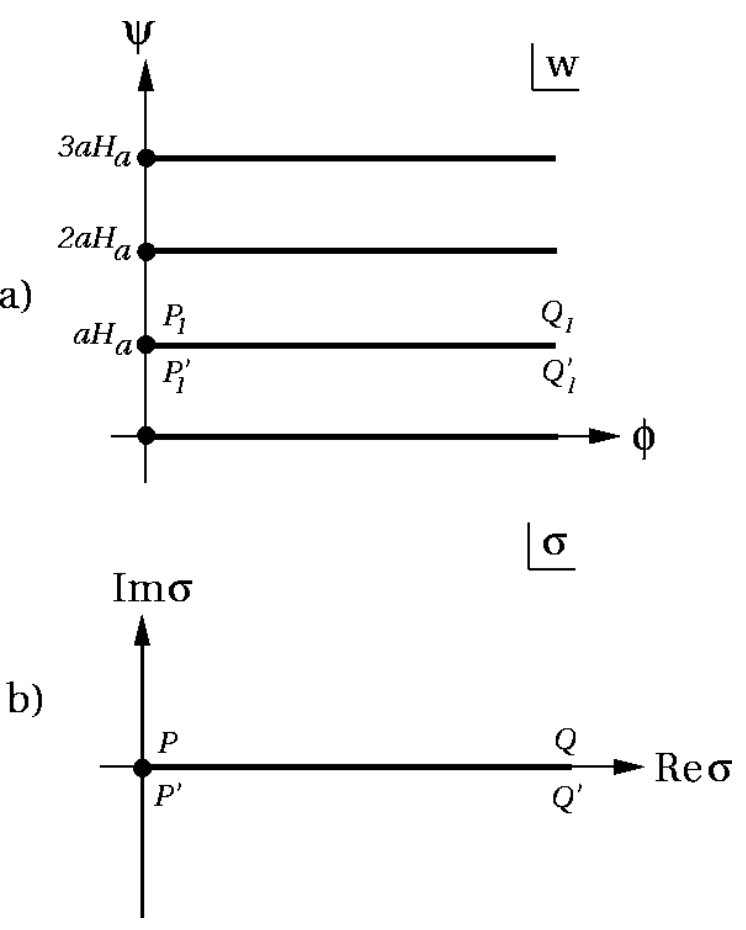

FIG. 4. The $w$ - and $\sigma$-planes.

On the interface we have $|\eta|=1$, and we define $\theta$ to be the tangent angle (as in Fig. 3) as $\eta=-e^{-i \theta}$ and only the phase $\theta$ changes as we traverse the interface from the point $P_{n}$ to $Q_{n}$; this produces a semi-circle in the $\eta$-plane. On the nose $\eta_{x}=0$, so that the segment $P_{n}^{\prime} P_{n}$ maps onto a horizontal straight line in the $\eta$ plane. Therefore, the region exterior to the superconducting laminae maps onto the interior of a semi-circle, as shown in Fig. 5(a) 
(the hodograph for the field).

We now need to map the $w$-plane onto the $\eta$-plane. This is most easily carried out by the following sequence of transformations. First, we take care of the periodicity in the $w$-plane by mapping it onto the $\sigma$-plane with the transformation

$$
\sigma=e^{2 \pi w / H_{a} a}-1,
$$

so that the $\sigma$-plane has a single cut along the positive real axis, as shown in Fig. $1(\mathrm{~b})$. Next, map the $\sigma$-plane onto the lower half of the $\lambda$-plane using

$$
\lambda=\left(\frac{k^{2}}{\sigma}\right)^{1 / 2}
$$

as shown in Fig. F(b). Here $k$ is a constant to be determined from the boundary conditions. The next step is to map the $\eta$-plane onto the $\lambda$-plane using the Joukowsky transformation

$$
\lambda=\frac{1}{2}\left(\frac{1}{\eta}+\eta\right) .
$$

To determine $k$, we notice that as $z \rightarrow \infty, w \rightarrow-\infty$, so that $\sigma=-1$ and $\eta=H_{a} / H_{n}$. Introducing the notation $h_{a} \equiv H_{a} / H_{n}$, we then have

$$
k^{2}=\frac{\left(1-h_{a}^{2}\right)^{2}}{4 h_{a}^{2}} .
$$

a)

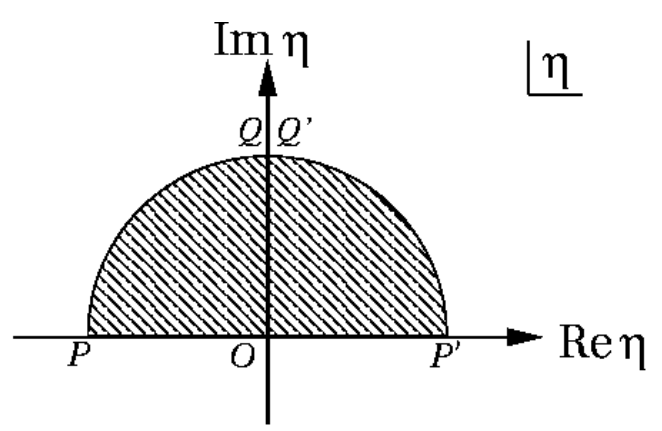

b)

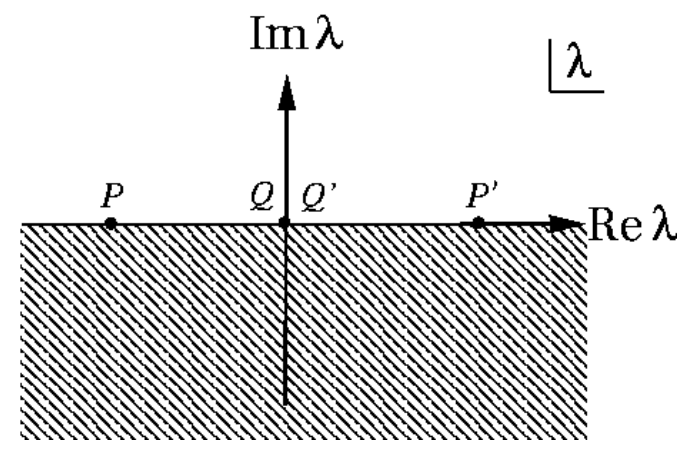

FIG. 5. The $\eta$ - and $\lambda$-planes.
Combining Eqs. (3.5)-(3.8), we have for the complex potential

$$
w=\frac{H_{a} a}{2 \pi} \ln \left[\frac{\left(\eta^{2}+h_{a}^{2}\right)\left(\eta^{2}+h_{a}^{-2}\right)}{\left(1+\eta^{2}\right)^{2}}\right] .
$$

To determine the shape of the lamina, we use Eq. (3.4) to write

$$
\begin{aligned}
\frac{d \zeta}{d \eta} & =\frac{d \zeta}{d w} \frac{d w}{d \eta}=-\frac{1}{H_{n}} \frac{1}{\eta} \frac{d w(\eta)}{d \eta} \\
& =-\frac{a}{\pi} \frac{\left(1-h_{a}^{2}\right)^{2}}{h_{a}} \frac{1-\eta^{2}}{\left(\eta^{2}+h_{a}^{-2}\right)\left(\eta^{2}+h_{a}^{2}\right)\left(\eta^{2}+1\right)} .
\end{aligned}
$$

The integral can be performed by using a partial fraction expansion. The constant of integration is determined by recognizing that when $\eta=0, z=0$. We finally have

$$
\begin{gathered}
\zeta(\eta)=-\frac{i a h_{a}}{\pi}\left[\frac{1}{2 h_{a}} \ln \left(\frac{h_{a}-i \eta}{h_{a}+i \eta}\right)+\frac{h_{a}}{2} \ln \left(\frac{h_{a}^{-1}-i \eta}{h_{a}^{-1}+i \eta}\right)\right. \\
\left.-\ln \left(\frac{1-i \eta}{1+i \eta}\right)\right] .
\end{gathered}
$$

This equation implicitly determines the magnetic field $\eta$ as a function of position $\zeta$. The particular parameterization is different from Landau's [7, 38], but can be shown to be equivalent. This result also appears to coincide with result of Fortini and Paumier [39], although the method of derivation is entirely different. The equivalent fluid problem would be the separated flow past a plate placed symmetrically in a channel; the solution to this problem is given in Ref. [37], p. 39, Eq. (25a), which is the same as Eq. (3.11) above.

To calculate the lamina shape, recall that on the normal-superconducting interface $\eta=-e^{-i \theta}$, with $\theta$ the tangent angle on the interface. Substituting into Eq. (3.11), and separating real and imaginary parts, after a great deal of algebra we obtain the following two parametric equations for the position of the interface:

$$
\begin{gathered}
x(\theta)=\frac{a}{2}\left[1-h_{a}-\frac{\left(1-h_{a}^{2}\right)}{\pi} \tan ^{-1}\left(\frac{2 h_{a} \cos \theta}{1-h_{a}^{2}}\right)\right] \\
z(\theta)=\frac{a}{4 \pi}\left[\left(1+h_{a}^{2}\right) \ln \left(\frac{1+h_{a}^{2}+2 h_{a} \sin \theta}{1+h_{a}^{2}-2 h_{a} \sin \theta}\right)\right. \\
\left.-4 h_{a} \ln \left(\frac{\cos \theta}{1-\sin \theta}\right)\right] .
\end{gathered}
$$

Using these parametric equations we can calculate the lamina shapes for different applied fields $h_{a}$; some representative results are given in Fig. 6. The width $2 b$ of the nose can now be determined from (3.12) by setting $\theta=0$, with the result

$$
b=\frac{a}{2}\left(1-h_{a}\right)-\frac{a}{2 \pi}\left(1-h_{a}^{2}\right) \sin ^{-1}\left(\frac{2 h_{a}}{1+h_{a}^{2}}\right) .
$$




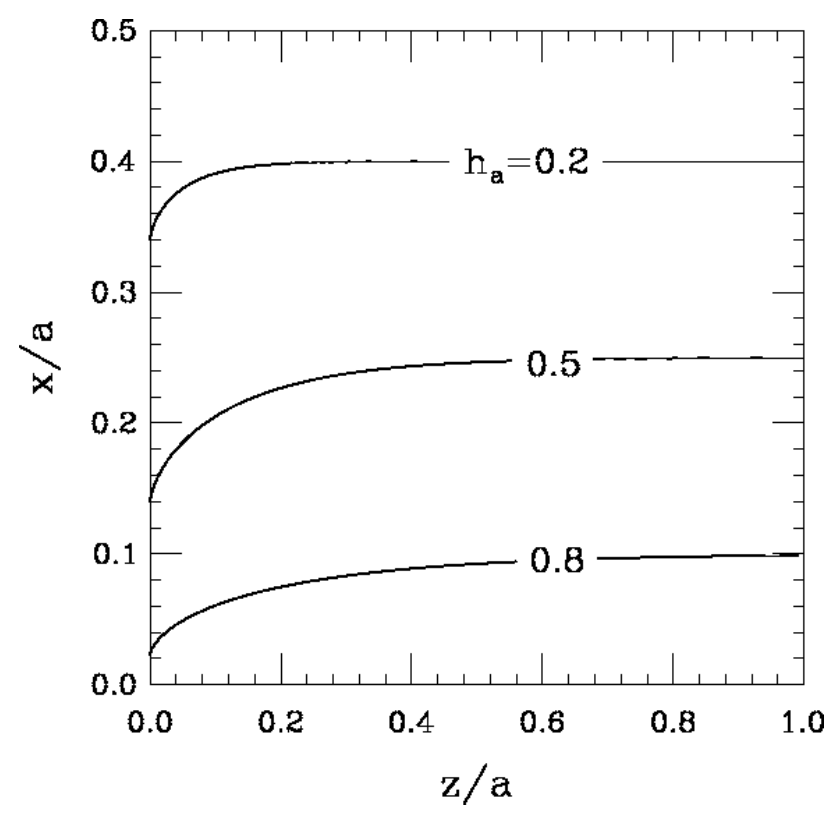

FIG. 6. Lamina shapes calculated from Eq. 3.12 for several values of the reduced field $h_{a}$.

\section{Energy of the laminar structure}

The total free energy of the laminar state has contributions from the condensation energy, the magnetic field, and the SN interfaces. The condensation energy is just $-H_{c}^{2} / 8 \pi$ times the volume occupied by the superconducting phase. If the normal-superconducting interfaces did not bend at all, this energy would be $-\left(H_{c}^{2} / 8 \pi\right)\left(N a_{s}\right) L_{y} d$, with $N$ the total number of laminae, $L_{y}$ the length of the sample in the $y$-direction, and $d$ the thickness of the sample [note that the sample has a total area $\left.A=(N a) L_{y}\right]$. To this we must add the condensation energy lost due to the thinning of the superconducting regions near the sample surfaces. The condensation energy per unit area is then

$$
\frac{E_{\mathrm{c}}}{A}=-\frac{H_{c}^{2} d}{8 \pi} \frac{a_{s}}{a}+\frac{4}{a} \frac{H_{c}^{2}}{8 \pi} \int_{-\infty}^{0}\left[\frac{a_{s}}{2}-x(z)\right] d z,
$$

where the factor of 4 in front of the integral accounts for the 4 corners of the lamina. The integral may be written as an integral over the tangent angle $\theta$ :

$$
\begin{aligned}
& \int_{-\infty}^{0}\left[\frac{a_{s}}{2}-x(z)\right] d z=-\int_{0}^{\pi / 2}\left[\frac{a_{s}}{2}-x(\theta)\right] \frac{d z}{d \theta} d \theta \\
= & \frac{a^{2} h_{a}\left(1-h_{a}^{2}\right)}{2 \pi^{2}} \int_{0}^{\pi / 2} d \theta \frac{\tan ^{-1}(p \cos \theta)\left(1-\cos ^{2} \theta\right)}{\cos \theta\left(1+p^{2} \cos ^{2} \theta\right)},
\end{aligned}
$$

where the second line was obtained by substituting from (3.12), and with $p=2 h_{a} /\left(1-h_{a}^{2}\right)$. After a partial fraction expansion and several integrations by parts the integral in 3.15$)$ is found to be

$$
\frac{\pi}{2}\left(\ln (p+q)-\frac{q}{p} \ln q\right)
$$

with $q=\left(1+p^{2}\right)^{1 / 2}$. We then have for the condensation energy

$$
\frac{E_{\mathrm{c}}}{A}=-\frac{H_{c}^{2} d}{8 \pi}+\frac{H_{c}^{2} d}{8 \pi}\left[\frac{a_{n}}{a}+\frac{a}{d} f_{c}\left(h_{a}\right)\right],
$$

with

$$
\begin{gathered}
f_{c}(h)=\frac{1-h^{2}}{2 \pi}\left[(1+h)^{2} \ln (1+h)+(1-h)^{2} \ln (1-h)\right. \\
\left.-\left(1+h^{2}\right) \ln \left(1+h^{2}\right)\right] .
\end{gathered}
$$

Next, we need to find the magnetic field energy, obtained by integrating $B^{2} / 8 \pi$ over all space, both inside and outside the sample. For our periodic structure the field energy per unit area becomes

$$
\frac{E_{\mathrm{m}}}{A}=\frac{2}{a} \int_{\mathcal{C}} \frac{B^{2}}{8 \pi} d x d z
$$

where the factor of 2 accounts for the top and bottom surfaces of the sample, and the integral is taken over the area $\mathcal{C}$ of one cell, as shown in Fig. 17. We can write $B^{2}=\left(\nabla A_{y}\right)^{2}$ in this two dimensional geometry, and then use the fact that $\nabla^{2} A_{y}=0$ to write Eq. (3.19) as a line integral around the boundary $\partial \mathcal{C}$ of the unit cell:

$$
\frac{E_{\mathrm{m}}}{A}=\frac{2}{8 \pi a} \int_{\partial \mathcal{C}} A_{y}(s) B_{s}(s) d s,
$$

with $A_{y}(s)$ the vector potential on the boundary, and $B_{s}(s)$ the component of the magnetic field which is tangent to the boundary.

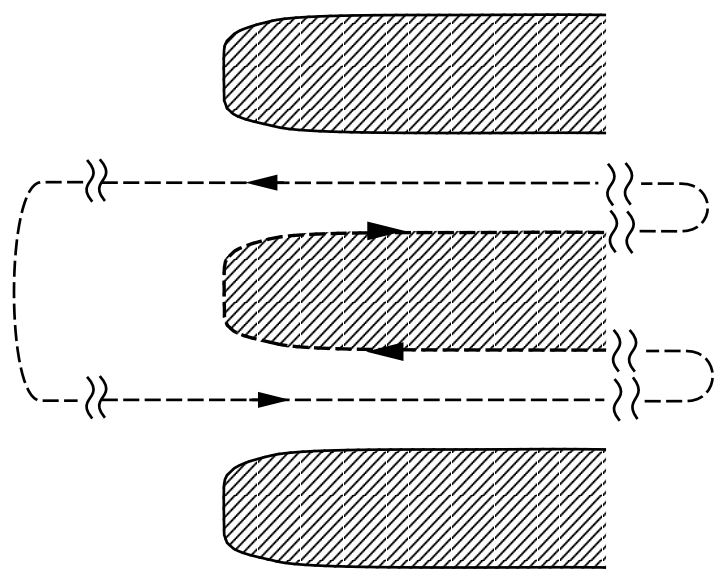

FIG. 7. Contour for energy calculation.

The advantage of this representation of the field energy is that the vector potential is constant on the boundaries, and it can therefore be brought outside the integral. Let's consider the various contributions to the integral. 
On the normal-superconducting interface (the segments $Q^{\prime} P^{\prime}$ and $\left.P Q\right)$ the integral $\int B_{s} d s$ vanishes, since the field points in the same direction on the left half of the superconducting lamina as on the right half. On the midline between the laminae labeled $n-1$ and $n$, the vector potential is $H_{a} a(n-1) / 2$ while on the next midline up it is $H_{a}(n+1) / 2$; the integral $\int B_{s} d s$ is equal in magnitude but opposite in sign for these two segments, since the integration paths are in opposite directions. Adding these two contributions, and using $B_{s}=-\partial \phi / \partial s$ we have

$$
\begin{aligned}
\frac{E_{\mathrm{m}}}{A} & =\frac{H_{a}}{4 \pi} \int_{\partial \mathcal{C}} B_{s} d s \\
& =\frac{H_{a}}{4 \pi}\left[\phi(a / 2,-d / 2)-\phi\left(a / 2, L_{z} / 2\right)\right],
\end{aligned}
$$

where $L_{z}$ is some large distance away from the top surface of the sample. The entire calculation of the field energy then reduces to finding the asymptotic behavior of the scalar potential along one of the streamlines (the midline, in this case). By examining the behavior of Eq. (3.9) for the complex potential $w$, and Eq. (3.11) for the position $\zeta$, as $\eta \rightarrow 1$ and $\eta \rightarrow h_{a}$, we find the asymptotic behavior $\phi\left(a / 2, L_{z} / 2\right) \sim-H_{a} L_{z} / 2+\phi_{+}$, where

$$
\begin{aligned}
& \phi_{+}=-\frac{H_{a} a}{2 \pi}\left[\ln 4+\left(1+h_{a}^{2}\right) \ln \left(1+h_{a}^{2}\right)\right. \\
& -\left(1+h_{a}\right)^{2} \ln \left(1+h_{a}\right) \\
& \left.-\left(1-h_{a}\right)^{2} \ln \left(1-h_{a}\right)\right] \text {, }
\end{aligned}
$$

and $\phi(a / 2,-d / 2) \sim-H_{n} a / 2+\phi_{-}$, where

$$
\begin{aligned}
\phi_{-}=-\frac{H_{n} a}{2 \pi}[ & \left(1-h_{a}\right)^{2} \ln \left(1-h_{a}\right) \\
& -\left(1+h_{a}\right)^{2} \ln \left(1+h_{a}\right) \\
& \left.+2 h_{a} \ln 4 h_{a}\right] .
\end{aligned}
$$

Substituting into 3.21), we have

$$
\frac{E_{\mathrm{m}}}{A}=\frac{H_{a}^{2}}{8 \pi} L_{z}+\frac{H_{n} H_{a}}{8 \pi} d+\frac{H_{n}^{2}}{4 \pi} a f_{\mathrm{mag}}\left(h_{a}\right),
$$

with

$$
\begin{aligned}
f_{\operatorname{mag}}\left(h_{a}\right)=\frac{h_{a}}{2 \pi}[ & \left(1+h_{a}\right)^{3} \ln \left(1+h_{a}\right) \\
& -\left(1-h_{a}\right)^{3} \ln \left(1-h_{a}\right) \\
& \left.-h_{a}\left(1+h_{a}^{2}\right) \ln \left(1+h_{a}^{2}\right)-2 h_{a} \ln 8 h_{a}\right] .
\end{aligned}
$$

The first term in Eq. (3.24) is the energy of the external field in the absence of the sample, which is of no interest and will be dropped from now on. The second term is the bulk magnetic field energy for a uniformly magnetized sample [40], and the third term is the energy arising from demagnetizing fields (due to the partitioning of the sample into domains).

Finally, we need to calculate the surface energy due to the normal-superconducting interfaces. If $\sigma_{S N}$ is the surface tension for the normal-superconducting interface, then the energy for a single interface is $\sigma_{S N} d L_{y}$ [41]. Since there are two interfaces per lamina, and $N$ lamina in the sample, the total energy due to the interfaces is $2 \sigma_{S N} d L_{y} N=2 \sigma_{S N} A(d / a)$. We can introduce a length $\Delta$, which is essentially the width of the interfaces, through $\sigma_{S N}=\left(H_{c}^{2} / 8 \pi\right) \Delta$; then the energy per unit area due to the interfaces is

$$
\frac{E_{\mathrm{int}}}{A}=\frac{H_{c}^{2}}{8 \pi} \frac{2 \Delta d}{a} .
$$

Adding together all of the contributions to the energy, Eqs. 3.17), (3.24), and (3.26), and using the flux conservation constraint $H_{n} a_{n}=H_{a} a$, we find

$$
\begin{aligned}
\frac{E}{A}= & \frac{H_{c}^{2} d}{8 \pi}\left\{-1+\left[\frac{a_{n}}{a}+h^{2} \frac{a}{a_{n}}\right]\right. \\
& \left.+2\left(\frac{\Delta}{a}+\frac{a}{d}\left[2 f_{\mathrm{c}}\left(h_{a}\right)+\frac{H_{n}^{2}}{H_{c}^{2}} f_{\mathrm{mag}}\left(h_{a}\right)\right]\right)\right\},
\end{aligned}
$$

with $h \equiv H_{a} / H_{c}$. The energy must be minimized with respect to both $a$ and $a_{n}$. This results in very cumbersome expressions. Instead, we will minimize the first term in brackets with respect to $a_{n}$, which yields $a_{n}=h a$, so that $H_{n}=H_{c}$ (and $h_{a}=h$ ). This is the result used by Landau, which is reasonably accurate as long as the surface and demagnetizing energies are small. If we substitute this back into the energy, we obtain

$$
\frac{E}{A}=-\frac{H_{c}^{2} d}{8 \pi}+\frac{H_{c} H_{a} d}{4 \pi}+\frac{H_{c}^{2} d}{4 \pi}\left[\frac{\Delta}{a}+\frac{a}{d} f_{\mathrm{L}}(h)\right] \text {, }
$$

with

$$
\begin{aligned}
f_{\mathrm{L}}(h)=2 f_{\mathrm{c}}(h)+f_{\operatorname{mag}}(h) & \\
=\frac{1}{4 \pi}[ & (1+h)^{4} \ln (1+h)+(1-h)^{4} \ln (1-h) \\
& \left.\quad-\left(1+h^{2}\right)^{2} \ln \left(1+h^{2}\right)-4 h^{2} \ln 8 h\right] .
\end{aligned}
$$

This function is plotted in Fig. 8. Its asymptotic behavior as $h \rightarrow 0$ is of interest in comparison with other approaches discussed below, and has the form

$$
f_{\mathrm{L}}(h) \simeq \frac{h^{2}}{\pi} \ln \left(\frac{0.56}{h}\right)
$$

Finally, the equilibrium laminar period is obtained simply by minimizing with respect to $a$, yielding

$$
a^{*}=\left[\frac{\Delta d}{f(h)}\right]^{1 / 2} \text {. }
$$




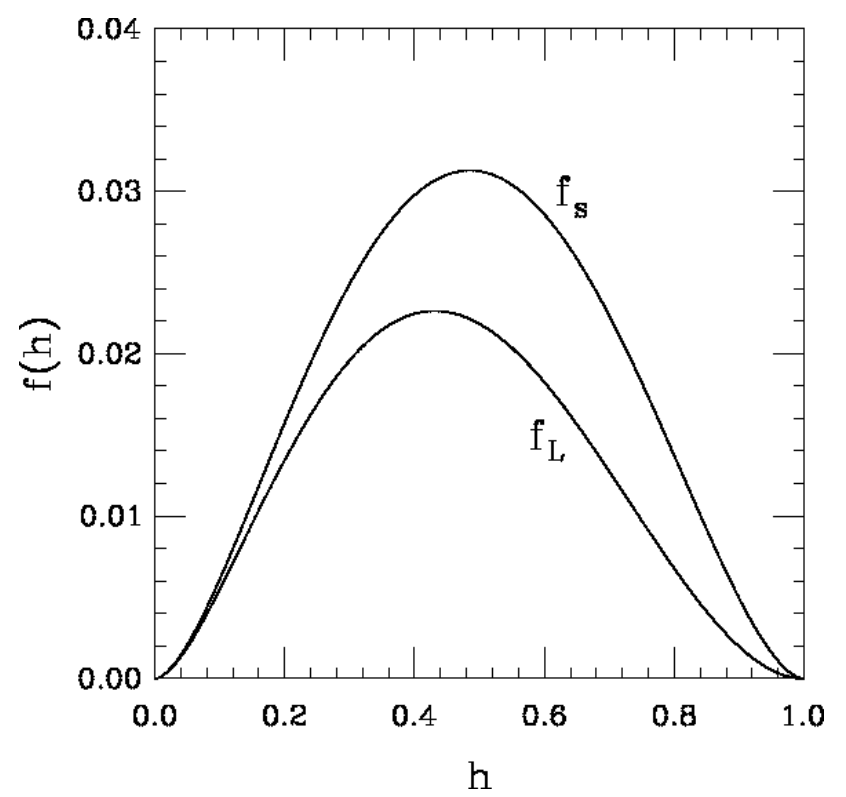

FIG. 8. The functions $f_{\mathrm{L}}(h)$ in Landau's model and $f_{s}(h)$ in the straight-lamina approximation.

This implies that the spacing diverges for small $h$ as

$$
a^{*} \simeq \frac{(\Delta d)^{1 / 2}}{h}\left[\frac{\pi}{\ln (0.56 / h)}\right]^{1 / 2}
$$

\section{B. Energy of a straight-lamina model}

It is useful to compare the results from the Landau model to an alternative straight-lamina model, which is illustrated in Fig. 9. In this model the field is still tangent to the normal-superconducting interface, but the magnitude of the field is not constant along the interface. The magnetic field and the complex potential can be obtained using standard conformal mapping methods; this problem is equivalent that of an ideal fluid flowing in a channel with an abrupt step [36]. The solution is

$$
\begin{gathered}
w=\frac{H_{a} a}{2 \pi} \ln \left(\frac{\eta^{2}+1}{\eta^{2}+h_{a}^{2}}\right), \\
\zeta=\frac{a_{s}}{2}+\frac{i a h_{a}}{2 \pi}\left[\ln \left(\frac{\eta+i}{\eta-i}\right)-\frac{1}{h_{a}} \ln \left(\frac{\eta+i h_{a}}{\eta-i h_{a}}\right)\right] .
\end{gathered}
$$

Using this solution it is possible to calculate the total energy, as in the Landau model. Making the simplifying assumption that $H_{n}=H_{c}$, we obtain

$$
\frac{E}{A}=-\frac{H_{c}^{2} d}{8 \pi}+\frac{H_{c} H_{a} d}{4 \pi}+\frac{H_{c}^{2} d}{4 \pi}\left[\frac{\Delta}{a}+\frac{a}{d} f_{s}(h)\right],
$$

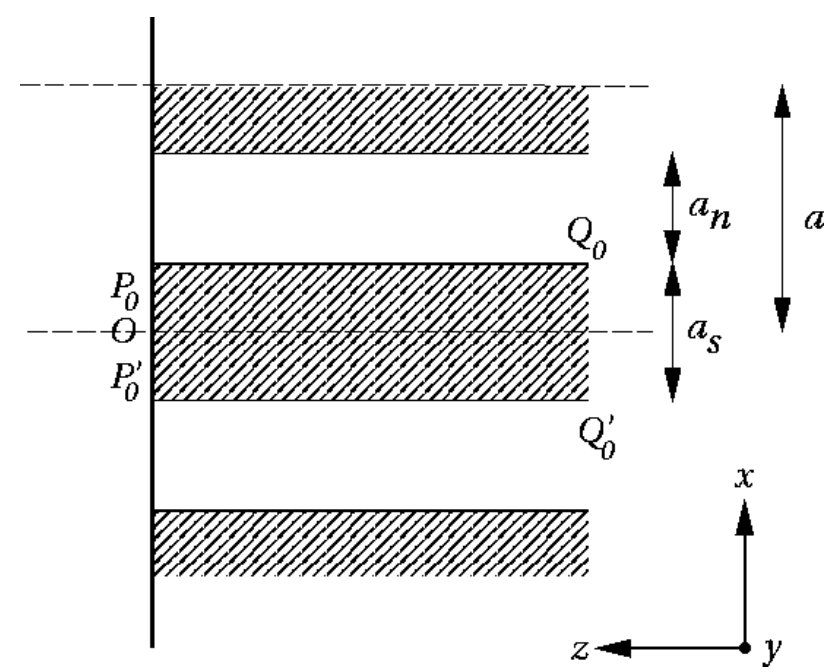

FIG. 9. Geometry of the straight-laminae model.

with

$$
\begin{gathered}
f_{s}(h)=\frac{h}{2 \pi}\left[(1+h)^{2} \ln (1+h)-(1-h)^{2} \ln (1-h)\right. \\
-2 h \ln 4 h] .
\end{gathered}
$$

This function is plotted in Fig. 8 for comparison with Landau's result (3.29). At small $h$ it behaves as

$$
f_{s}(h) \simeq \frac{h^{2}}{\pi} \ln \left(\frac{0.68}{h}\right)
$$

which is very close to the Landau result (3.30). We see that the total energy for this model has the same qualitative dependence upon the lamina spacing $a$ as the Landau model, although the Landau model has a lower energy for any reduced field $h$.

\section{Laminae shapes in finite-thickness plates}

In the analyses above we have assumed that the slab of superconductor is sufficiently thick that the shapes of the lamina walls can be computed as for a semi-infinite slab. When the thickness $d$ becomes small enough the wall shapes will change. From the asymptotic behavior of the semi-infinite solutions (3.12), we deduce that the thickness approaches its asymptotic values for $z \rightarrow-\infty$ as

$$
\frac{a}{2}\left(1-h_{a}\right)-x(z) \sim \frac{4 h_{a}}{\pi} \exp \left(\pi z / a h_{a}\right) .
$$

The decay length $a h_{a} / \pi$ should then determine when finite-slab thickness effects become important. The asymptotic results (3.30) and (3.37) show that the product $a h$ vanishes very slowly (logarithmically) as $h \rightarrow 0$, so that while such finite-thickness effects become important in that limit, practically the relevant fields are very 
small. From the asymptotics, the crossover field $h_{x}$ for $a h_{a} / \pi=d$ is on the order of

$$
h_{x} \sim 0.56 \exp (-\Delta / \pi d)
$$

For $h \leq h_{x}$ the slab thickness has no significant effect on the domain wall shapes. In the fluid dynamical analogy, the finite-thickness slab calculation is equivalent to so-called Riabouchinsky flows around two plates, the details of which the interested reader will find in standard references 37.

\section{THE CURRENT LOOP MODEL}

The analysis of the laminar state in the previous section has shown that in accounting for the flaring of the normal domains Landau's free-boundary approach yields a lower energy structure than a model with straight walls. But the analytical and numerical differences between the two approaches are relatively minor. In both models the supercurrents flow along the SN interfaces and on the top and bottom surfaces of the sample [18,42]. Just as the magnetic field in a solenoid is more nearly uniform when it is a tall thin cylinder than when it is short and wide, so too do we expect that the contributions from circulating currents along the SN interfaces will dominate when the flux domains are narrow and tall, at low applied fields $h$. This suggests that the basic physics of the laminar state can be understood by considering those circulating currents alone. We develop this current-loop model in the present section and show that it rather accurately reproduces the results of Landau's model. This calibration is an important test of an approach that can easily be generalized to SN interfaces of arbitrary shape.

\section{A. Energetics of the current loop model}

For the purposes of this model, the intermediate state is described in macroscopic terms. As above, it has thickness $d$, total area $A$, and volume $V=A d$, but now the SN interfaces encircling each of the normal regions belong to a set $\left\{\mathcal{D}_{i}\right\}$ each with area $A_{i}$ and perimeter $L_{i}$. The two phases occupy volumes $V_{s}$ and $V_{n}=d \sum_{i} A_{i}$, with $V_{s}+V_{n}=V$. Parameterizing each boundary by arclength, the position vectors of the interfaces are $\mathbf{r}_{i}(s)$. As in the straight-lamina model, we assume that $\mathbf{r}_{i}$ is independent of $z$, neglecting the flaring of the domain walls near the film surfaces that is seen in Fig. 6 .

The total energy of the system is as before a sum of the condensation energy $E_{c}$, the interfacial energy $E_{\text {int }}$, and the magnetic field energy $E_{\mathrm{m}}$,

$$
E\left[\left\{\mathbf{r}_{i}\right\}\right]=E_{c}+E_{\mathrm{int}}+E_{\mathrm{m}} .
$$

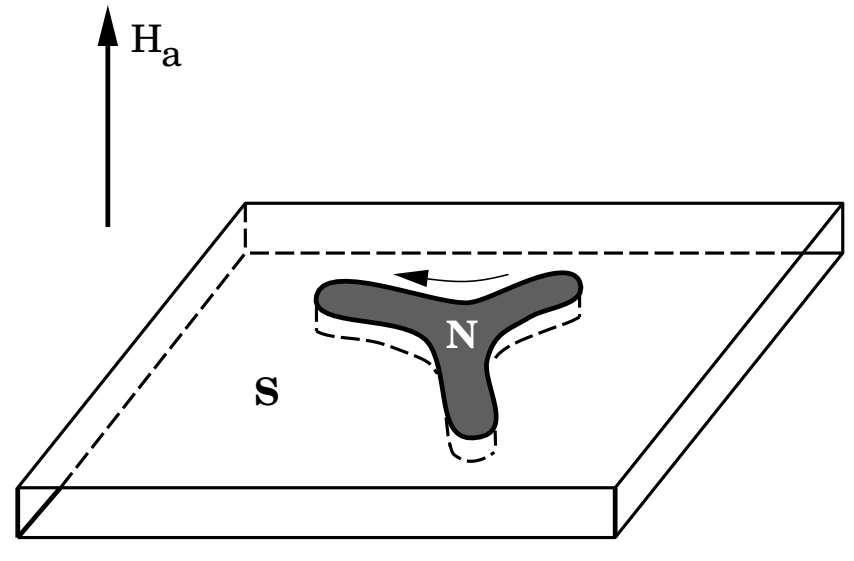

FIG. 10. A current loop.

And as before,

$$
E_{\mathrm{c}}=V \frac{H_{c}^{2}}{8 \pi} \rho_{n}, \quad E_{\mathrm{int}}=\frac{H_{c}^{2}}{8 \pi} \Delta d \sum_{i} L_{i},
$$

where $\rho_{n}=A_{n} / A$ is the area fraction for the normal phase, and where $E_{\mathrm{c}}$ is measured with respect to the purely superconducting state. Global flux conservation relates the field in the normal regions to the applied field, $H_{n}=H_{a} / \rho_{n}$, and by the tangential continuity of $\mathbf{H}$ across a S-N interface the field in the superconducting region is $H_{s}=H_{n}$. The superconducting regions are perfectly diamagnetic; they each have a magnetization $\mathbf{M}=-\left(H_{n} / 4 \pi\right) \hat{\mathbf{e}}_{z}$, related in the usual way to the (Meissner) currents that flow along the SN (and top and bottom sample) boundaries.

We compute the magnetic field energy as a sum of two contributions, the first of which is that of the domain magnetizations $\mathbf{M}$ in the presence of the external field $\left(-\int d^{3} r \mathbf{H}_{a} \cdot \mathbf{M}\right)$. The second contribution is the selfand mutual-induction of the current ribbons. Expressing these in terms of the macroscopic quantities $\rho_{n}$, etc., and the current-ribbon positions we have

$$
\begin{aligned}
E_{\mathrm{m}}= & V \frac{H_{a} H_{n}}{4 \pi}\left(1-\rho_{n}\right) \\
& -\frac{1}{2} M^{2} \sum_{i, j} \int_{0}^{d} d z \int_{0}^{d} d z^{\prime} \oint d s \oint d s^{\prime} \frac{\hat{\mathbf{t}}_{i} \cdot \hat{\mathbf{t}}_{j}}{R_{i j}},
\end{aligned}
$$

where $M=-H_{n} / 4 \pi$. Here, the vectors $\hat{\mathbf{t}}_{i}=\hat{\mathbf{t}}_{i}(s)$ are unit tangents to the current ribbons and label the direction of the current flow. By the usual screening processes in superconductors, the direction of the flow is so as to cancel the applied field in the superconducting regions and augment it in the normal regions (see Fig. 10). The scalar product of the tangent vectors is however invariant under the overall reversal of the parameterizations $(s \rightarrow-s)$. The current-current interaction is Coulombic, with $R_{i j}=\left\{\left[\mathbf{r}_{i}(s)-\mathbf{r}_{j}\left(s^{\prime}\right)\right]^{2}+\left(z-z^{\prime}\right)^{2}\right\}^{1 / 2}$. While the $z$ and $z^{\prime}$ integrals are readily performed (see below), the more elementary form (4.3) serves to remind us that the 
current-current interactions are in their free-space form. By performing the $z$ and $z^{\prime}$ integrals, the magnetic field energy becomes

$$
\begin{aligned}
E_{\mathrm{m}}= & V \frac{H_{a} H_{n}}{4 \pi}\left(1-\rho_{n}\right) \\
& -M^{2} d \sum_{i, j} \oint d s \oint d s^{\prime} \hat{\mathbf{t}}_{i} \cdot \hat{\mathbf{t}}_{j} \Phi\left(R_{i j} / d\right)
\end{aligned}
$$

where now the elementary free boundaries are contours in the plane, interacting with the potential

$$
\begin{aligned}
\Phi(R / d) & =\frac{1}{2 d} \int_{0}^{d} d z \int_{0}^{d} d z^{\prime}\left[R^{2}+\left(z-z^{\prime}\right)^{2}\right]^{-1 / 2} \\
& =\sinh ^{-1}(d / R)+R / d-\sqrt{1+(R / d)^{2}}
\end{aligned}
$$

where $R=|\mathbf{R}|$ with $\mathbf{R}\left(s, s^{\prime}\right)=\mathbf{r}(s)-\mathbf{r}\left(s^{\prime}\right)$ the in-plane vector between points labeled by $s$ and $s^{\prime}$. As discussed elsewhere 23] this potential is Coulombic for $R \gg d$, $\Phi \approx d /(2 R)$, but for $R \ll d, \Phi \approx \ln \left(2 e^{-1} d / R\right)$, with the film thickness $d$ acting as a cutoff. Note the interesting parallel with Pearl's interaction potential (2.17) among vortices in thin films. The fact that this interaction potential is in some cases identical and in others nearly identical to that found in the free-boundary approach to a number of other systems offers an explanation for the similarity in their behavior. Table II summarizes these analogies between the different systems.

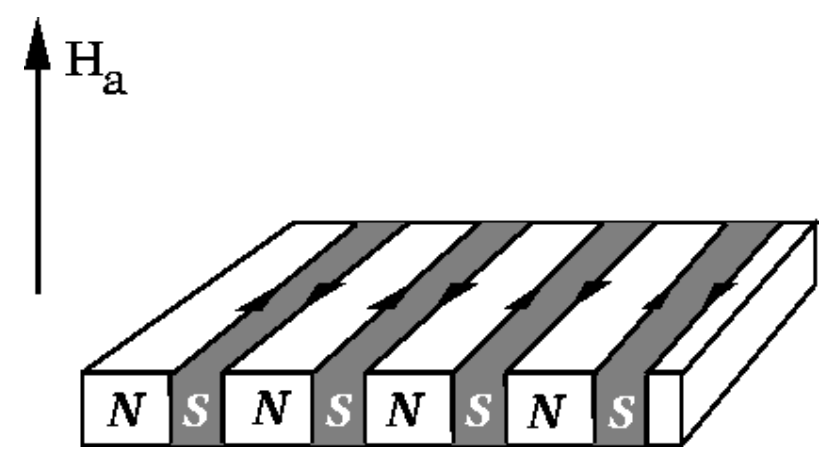

FIG. 11. The laminar state as a collection of current loops. Arrows indicate directions of the current, tangential to the $\mathrm{SN}$ interfaces.

\section{B. Current-loop description of the laminar state}

Now we calculate the energy of the laminar state in the CL model. As before, the periodicity length in the plane is $a$, and the width of the normal lamina is $a_{n}$; we will assume that the pattern is periodic in the $x$-direction. The nonlocal magnetic contribution is the only difficult one, and it proves more convenient to return to the original self-induction form of the tangent vector coupling, before averaging over the slab thickness. It is then easy to introduce a Fourier representation, which for a uniform laminar structure yields the magnetic field energy $E_{\mathrm{m}}$ per unit area

$$
\begin{aligned}
\frac{E_{\mathrm{m}}}{A}= & -\frac{M^{2}}{a} \sum_{n=-\infty}^{\infty} \int_{-\infty}^{\infty} d y \int_{0}^{d} d z \int_{0}^{d} d z^{\prime} \\
& \times \int \frac{d^{3} q}{(2 \pi)^{3}} \frac{4 \pi}{q^{2}} e^{i\left[n q_{x} a+q_{y} y+q_{z}\left(z-z^{\prime}\right)\right]}\left(1-e^{i q_{x} a_{n}}\right) .
\end{aligned}
$$

Several straightforward integrations reduce this to

$$
\begin{aligned}
\frac{E_{\mathrm{m}}}{A}=-\frac{a M^{2}}{\pi^{2}} \sum_{n=1}^{\infty} & \frac{\left[1-\cos \left(2 \pi n a_{n} / a\right)\right]}{n^{3}} \\
& \times\left(\frac{2 \pi n d}{a}+e^{-2 \pi d n / a}-1\right) .
\end{aligned}
$$

Note that the last term contains all of the $d$-dependence of the sum. The leading contribution in the limit of large slab thickness is a "bulk" contribution expressible simply in terms of the stripe dimensions

$$
\sum_{n=1}^{\infty} \frac{\left[1-\cos \left(2 \pi n a_{n} / a\right)\right]}{n^{2}}=\pi^{2} \frac{a_{n}\left(a-a_{n}\right)}{a^{2}} .
$$

It follows that the form of the free energy is exactly like that in Eqs. (3.27) and 3.28 ), but with a new function $f$,

$$
f_{\mathrm{CL}}(h, d, a)=\frac{1}{2 \pi^{3}} \sum_{n=1}^{\infty} \frac{\sin ^{2}(n \pi h)}{n^{3}}\left[1-e^{-2 \pi n d / a}\right] .
$$

As alluded to in the discussion of the laminar shapes in Landau's calculation, finite-thickness effects show up when $d$ is comparable to $a$.

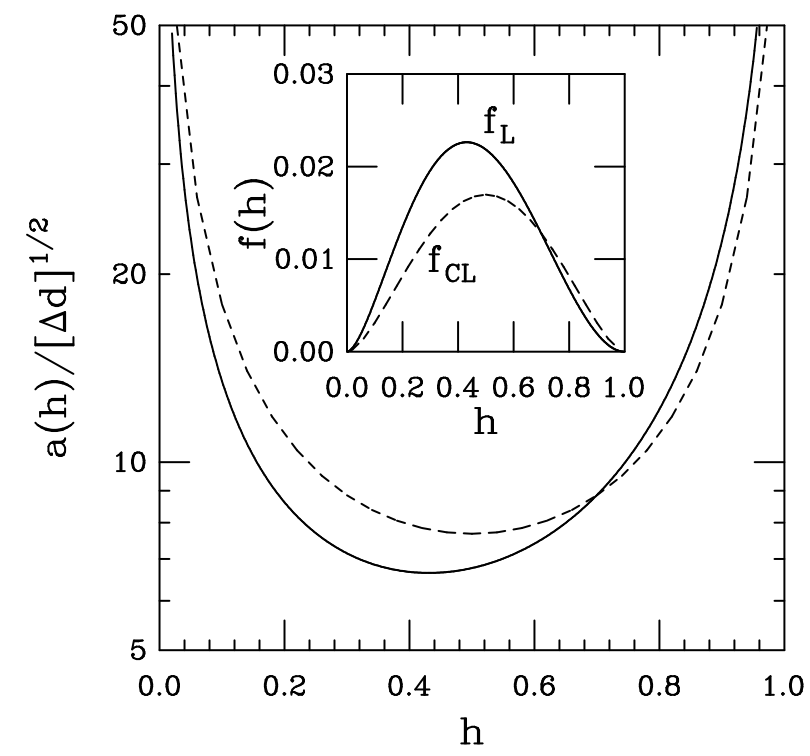

FIG. 12. Comparison of current loop model and Landau model for laminar state. Equilibrium laminar spacing $a(h)$ for Landau model (solid) and current-loop model (dashed). Inset shows the functions $f_{L}$ (solid) and $f_{C L}$ (dashed). 
There are several noteworthy features of the function $f_{\mathrm{CL}}(h)$, particularly in the limit $d / a \gg 1$ which we consider here. First, as shown in Fig. 12, it is rather close to the Landau function, and hence its implications for the equilibrium laminar thickness are in reasonable accord with experiment. Second, analytically it has the same structure as $f_{\mathrm{L}}$ as $h \rightarrow 0$,

$$
f_{\mathrm{CL}}(h) \simeq \frac{h^{2}}{2 \pi} \ln \left(\frac{0.71}{h}\right) .
$$

Third, it has an exact symmetry under the transformation $h \rightarrow 1-h$, a reflection in part of the straight SN interfaces presumed in the model. This symmetry is absent in Landau's calculation and in the straight-lamina model by virtue of the currents on the slab faces. Finally, the form of the magnetic field energy in Eq. (4.7) is identical to the field energy of a stripe array in a thin ferromagnetic film 43,44 .

\section{A dynamical model}

The energetic competition between surface tension and self-induction present in the current-loop model appears in a number of other contexts (Table II), where it has been shown to produce also a rich dynamical behavior. While the precise connection between the Young-Laplace and Biot-Savart forces and the interface dynamics depends on the physical setting (e.g. Hele-Shaw flow with Darcy's Law, surfactant monolayers at the air-water interface with coupling to the fluid phase, reaction-diffusion systems), the phenomenon of repeated branching instabilities producing disordered lamellar structures is ubiquitous. This suggests that much can be learned by studying the very simplest dynamical law for interface motion, the local dissipation model [23], in which a local viscous drag acting at the interface balances the local pressure difference, the latter computed as a functional derivative as in Eq. (2.7).

As a first step toward a full study of the many-interface current-loop model of the intermediate state, we study here the simplest mean-field description of a single current loop. That loop is assigned to a cell (analogous to a Wigner-Seitz cell) of area $A_{\text {cell }}$ from which we compute the area fraction $\rho_{n}=A_{n} / A_{\text {cell }}$. In this approximate description, the self-induction of the loop is retained in its full form, but the mutual induction term in the energy associated with all other loops only contributes a bulk energy term like that seen in the laminar calculation (4.8). Moreover, the amplitude of the circulating currents is taken to be set by $H_{c}$ rather than the actual local field. This is equivalent to assuming that the actual area fraction departs little from its equilibrium value. The system is then characterized by a single geometrical parameter $p \equiv 2 R_{\text {equiv }} / d$ describing the aspect ratio, where $R_{\text {equiv }}$ is the radius of the circle whose area is that of the initial condition, and a single energetic parameter, the reduced magnetic field $h$. All other parameters simply rescale time.

Within this model, the normal component of the interface velocity is found by functional differentiation:

$$
\begin{aligned}
\hat{\mathbf{n}} \cdot \mathbf{r}_{t}(s)=\frac{H_{c}^{2} d}{8 \pi \eta} & \{\Pi-\Delta \mathcal{K}(s) \\
& \left.-\frac{1}{2 \pi d} \oint d s^{\prime} \hat{\mathbf{R}} \times \hat{\mathbf{t}}\left(s^{\prime}\right) \Psi(R / d)\right\},
\end{aligned}
$$

with $\mathcal{K}(s)$ the curvature, and $\Psi(\xi)=\Phi^{\prime}(\xi)=1-(1+$ $\left.\xi^{-2}\right)^{1 / 2}$ is the generalization of the Coulombic form of the Biot-Savart force to finite-thickness slabs [23]. Finally, the pressure term is

$$
\Pi=h^{2} / \rho_{n}^{2}-1 .
$$

The kinetic coefficient $\eta$ may be estimated [13] from results on the bulk properties of strongly type-I systems,

$$
\eta=\frac{H_{c}^{2} d \Delta}{8 \pi} \frac{\pi \hbar}{8 k_{B} T_{c} \xi_{0}^{2}},
$$

where again $T_{c}$ is the critical temperature and $\xi_{0}$ is the bare correlation length 45.

A contour dynamics such as (4.11) is readily generalized to account for surface tension anisotropy, a material feature that has long been suggested to play a role in the morphology of the intermediate state patterns [1], as it does in problems such as dendritic growth [46]. When the anisotropy is $q$-fold, the parameter $\Delta$ has the form

$$
\Delta=\Delta_{0}[1+\epsilon \cos (q \theta)] .
$$

Typical experiments show a $q=4$ or $q=6$ anisotropy [47]. Our intuition suggests that the variation of $\sigma_{S N}$ through $\Delta$ will bias instabilities toward $q$-fold symmetry, and lead to preferred orientations of flux stripes produced from those instabilities.

\section{Instabilities; numerical studies}

Two regular geometries of flux domains have historically been of interest: circles and stripes. In the next section we consider in detail the stability of stripes and stripe arrays (the laminar state); here we focus on fingering and branching instabilities of circular domains. Since linear stability analyses for circular interfaces have been presented in detail elsewhere in the context of closely related models 22 24, 26, 27,30, we will not repeat them here in detail. Two important qualitative results from those studies are that for a given size (domain radius and slab thickness) (i) there exists a critical applied field below which the circle is stable and above which azimuthal 
modes become active, and (ii) instabilities of increasing mode number occur with ever larger applied field. These properties may be illustrated through numerical studies of the contour dynamics, which allow us to see the highly nonlinear regime far beyond the instabilities.

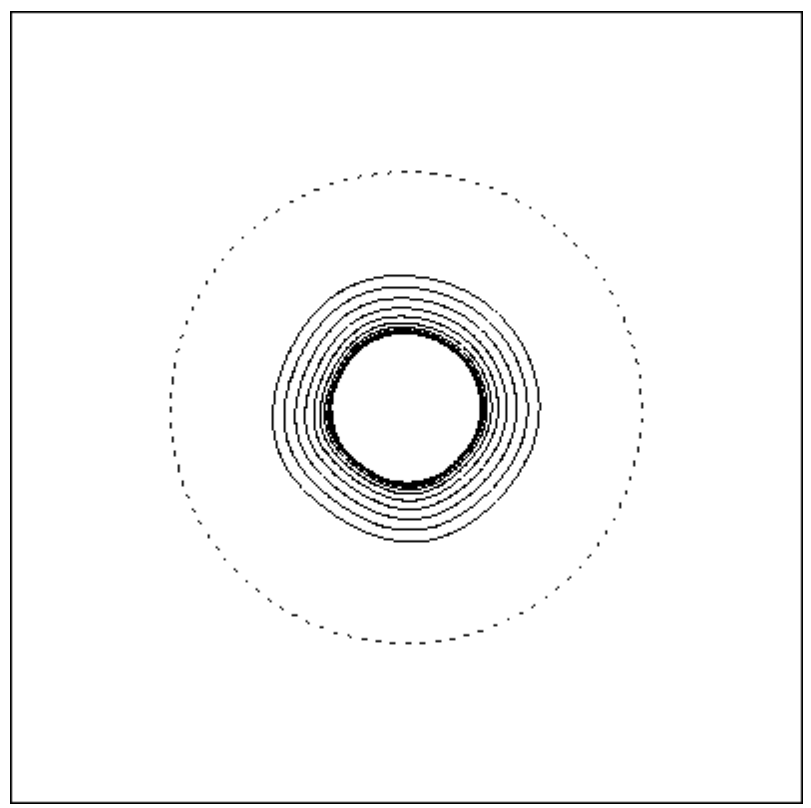

FIG. 13. Results from numerical studies of the current-loop model. Relaxation of a circular initial condition to a stable circular final state of different radius. Dashed line shows the area of the unit cell. Parameters are $p=5.0$ and $h=0.1$.

An efficient numerical method for studying this nonlocal interface dynamics has been described in considerable detail elsewhere [27:30]. It uses pseudo-spectral techniques to solve for the time evolution of the local tangent angle $\theta(s)$, from which the $(x(s), y(s))$ coordinates of the interface are computed by basic differential geometry. For the purposes of verifying the analytical stability results as well as investigating such phenomena as mode competition, the initial contour is given a curvature $\mathcal{K}$ perturbed from that of a circle,

$$
\mathcal{K}(\alpha)=\frac{1}{R_{0}}+\sum_{n=2}\left[a_{n} \cos (n \alpha)+b_{n} \sin (n \alpha)\right],
$$

where $R_{0}$ is the unperturbed radius and $\alpha=s / R_{0}$.

There are three basic phenomena that may be illustrated with the contour dynamics. The first, shown in Fig. 13, is the relaxation of a weakly perturbed circular domain whose initial area fraction is not the equilibrium value. This stable relaxation to a circle can occur if the applied field $h$ is below the instability value at the aspect ratio of interest. The asymptotic area fraction at long-times is $\rho_{n} \simeq h$, apart from a small correction due to surface tension. The approach of $\rho_{n}$ to this limiting value is shown in Fig. 14.

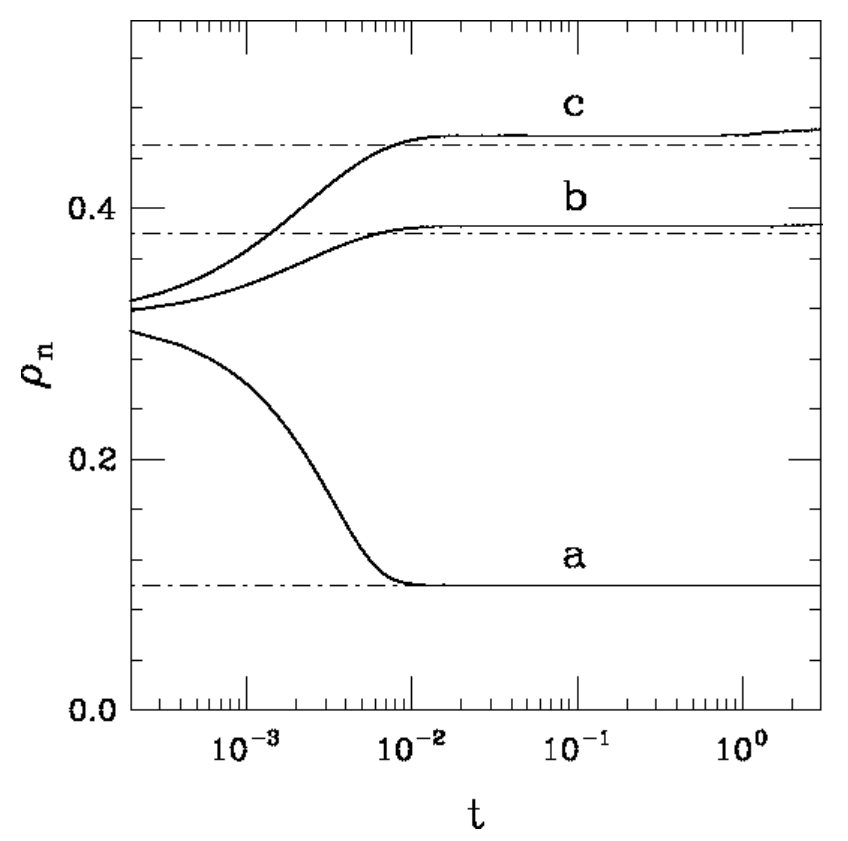

FIG. 14. Time evolution of the normal area fraction $\rho_{n}$ for a single domain governed by the contour dynamics (4.11). Curves (a), (b), and (c) correspond to Figs. 13, 15, and 16. Dashed lines indicate the relation $\rho_{n}=h$ determined by the bulk energetic contributions alone.

A second phenomenon occurs at higher $h$, and is the elementary elongational instability of a circular flux domain, as illustrated in Fig. 14. Again the area fraction evolves toward $\rho_{n}=h$, but now the deviation is significant due to larger contributions from the Biot-Savart integral. This shape evolution shows one the means by which finite stripes may form in the intermediate state.

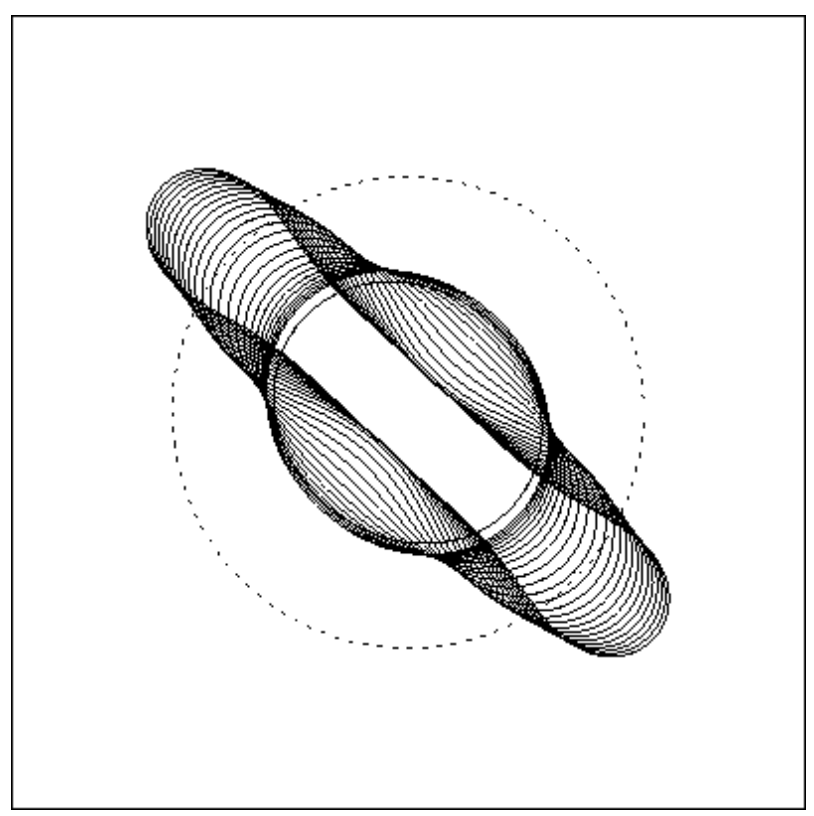

FIG. 15. Elongational instability of a circular flux domain, with aspect ratio as in Fig. 13 , but $h=0.38$. 
The curious feature of bulbous tips to the stripe is a rather common observation in dipolar systems. It is suggestive that the instability is in some sense related to evolution toward the fissioning of the original circle into two smaller ones. Energetic arguments based on this picture show that it rather accurately predicts the onset of this instability [27].

The third phenomenon of interest is the branching instability that occurs for still higher values of $h$, as shown in Fig. 15. The initial condition for this simulation was a circle perturbed with a small amplitude mode of azimuthal number 3. Rapid growth of that mode is followed by relaxation to "arms" of rather uniform width. The angles of the three "arms" forming the vertex are close to $120^{\circ}$, as is typical in systems governed by surface tension.

We conclude from these studies that a physical mechanism to produce the branched and fingered stationary shapes of flux domains in the intermediate state is the mechanical instability illustrated in Figures 15 and 16 .

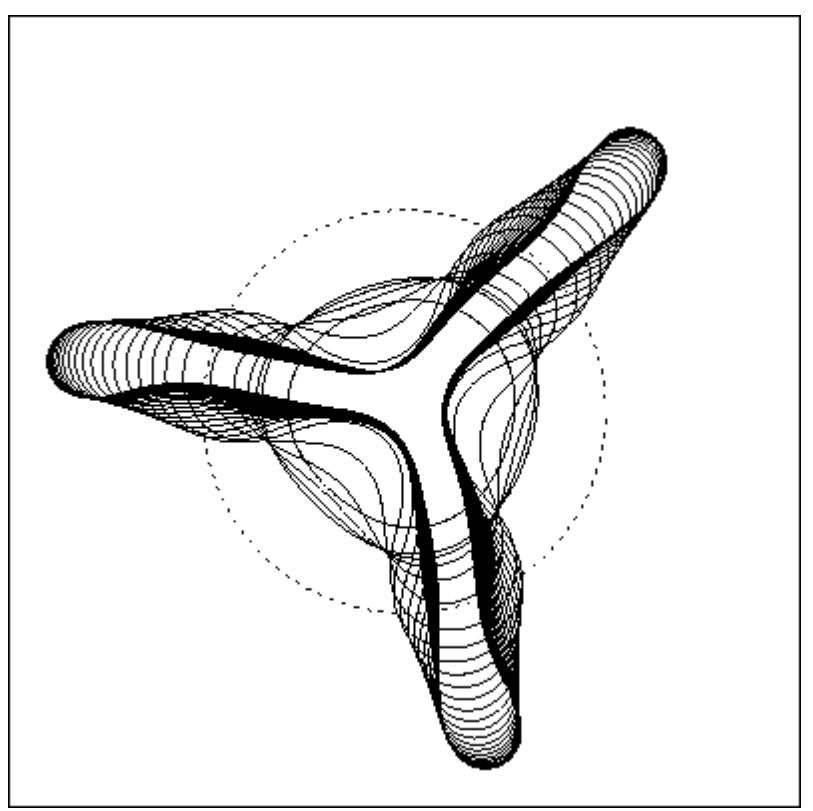

FIG. 16. Numerical study of branching instability, with $h=0.45$.

Finally, Fig. 17 shows the effects of surface tension anisotropy on the branching instability of the same initial condition as in Fig. 16. While the time evolution first produces a four-fold vertex, it subsequently fissions into two three-fold vertices that move away from each other. The branches of the pattern have oriented themselves with respect to the low-tension directions determined by the anisotropy (indicated by arrows). The instability of vertices of higher order than three is a common feature of dipolar systems.

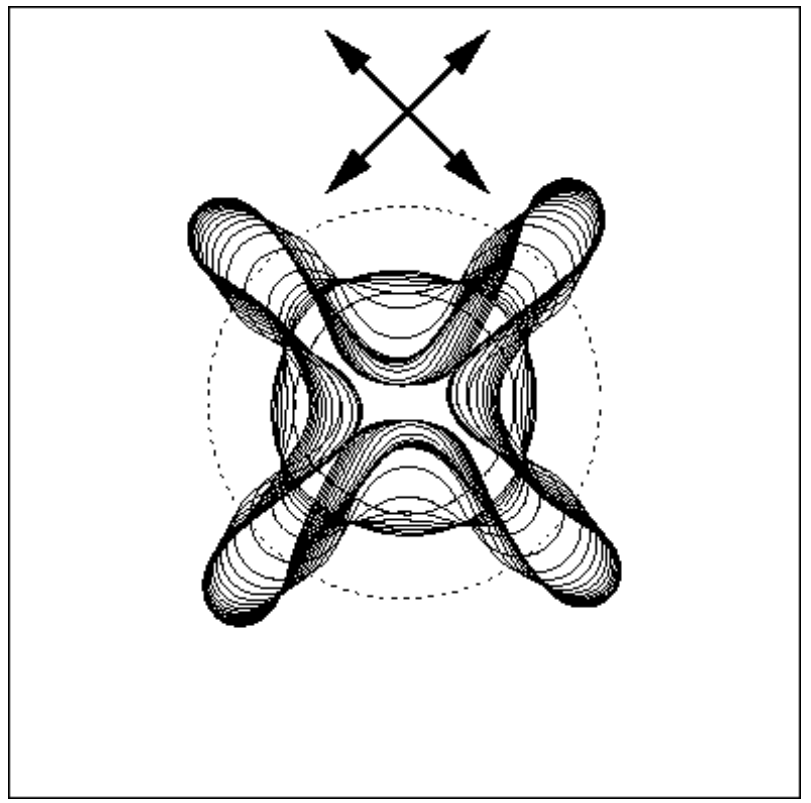

FIG. 17. Numerical study of branching instability, with $h=0.45$ and surface tension anisotropy $(\epsilon=0.1, q=4)$. Arrows indicate easy axes of low surface tension.

\section{PERTURBATIONS AROUND THE LAMINAR STATE}

In the absence of any in-plane component to the applied magnetic field, flux domains in the intermediate state often have the shape of buckled lamina. The wavelength is typically larger than the stripe width, as is the amplitude of the modulation. The conformal mapping algorithm for the laminar state is not generalizable to treat such truly three-dimensional structures, and there does not appear to have been any stability calculation of the laminar state. In the following sections we compute both the stability and elastic properties of flux stripes, making connections with pattern forming properties in other systems.

\section{A. Energy and stability of a single flux stripe}

Consider first the properties of a single flux stripe as described by the current-loop model. If the stripe has width $w$ in the $x$-direction, a length $l$ in the $y$-direction, and the plate spacing is $d$, then by considering the selfand mutual induction of the currents flowing along the edges the reduced energy $\tilde{E} \equiv E / 2 \sigma_{S N} A$ per unit area is

$$
\tilde{E}=\frac{1}{\alpha}-\frac{N_{B}}{\alpha} \int_{0}^{\infty} d \xi\left[\Phi(\xi)-\Phi\left(\sqrt{\xi^{2}+\alpha^{2}}\right)\right]
$$

where $\alpha=w / d$ and $N_{B}=2 M^{2} d / \sigma_{S N}$ is the dimensionless magnetic Bond number. The integrals are standard and yield the result 


$$
\begin{aligned}
\tilde{E}=\frac{1}{\alpha} & +\frac{N_{B}}{4 \alpha}\left[\alpha^{2} \ln \left(1+\alpha^{-2}\right)\right. \\
& \left.+4 \alpha \tan ^{-1} \alpha-\ln \left(1+\alpha^{2}\right)\right] .
\end{aligned}
$$

Figure 18 shows the stripe energy as a function of its width for various Bond numbers. We see that the minimum of this energy becomes sharper as $N_{B}$ increases. Minimizing $\tilde{E}$ with respect to $\alpha$ (hence with respect to the width) at fixed area $A$ yields a relation between $\alpha$ and $N_{B}$,

$$
1-\frac{N_{B}}{4}\left[\alpha^{2} \ln \left(1+\alpha^{-2}\right)+\ln \left(1+\alpha^{2}\right)\right]=0 .
$$

Now we connect this result to the stability analysis of the stripe. As shown in Fig. 19(a) and (b) there are two classes of small distortions we must consider. The first ("peristaltic") involves antisymmetric perturbations and changes the local stripe width. This will be of higher energy than the symmetric (or "serpentine") distortions of Fig. 19(b) which preserve the width. It is most convenient to calculate the linearized force acting on the interface, for which we use the result quoted in Eq. (4.11), written out explicitly,

$$
\begin{aligned}
-\gamma \mathcal{K}+2 M^{2} \oint d s^{\prime} & \frac{\left(\mathbf{r}(s)-\mathbf{r}\left(s^{\prime}\right)\right)}{\left|\mathbf{r}(s)-\mathbf{r}\left(s^{\prime}\right)\right|} \times \hat{\mathbf{t}}\left(s^{\prime}\right) \\
& \times\left\{\sqrt{1+\frac{d^{2}}{\left|\mathbf{r}(s)-\mathbf{r}\left(s^{\prime}\right)\right|^{2}}}-1\right\} .
\end{aligned}
$$

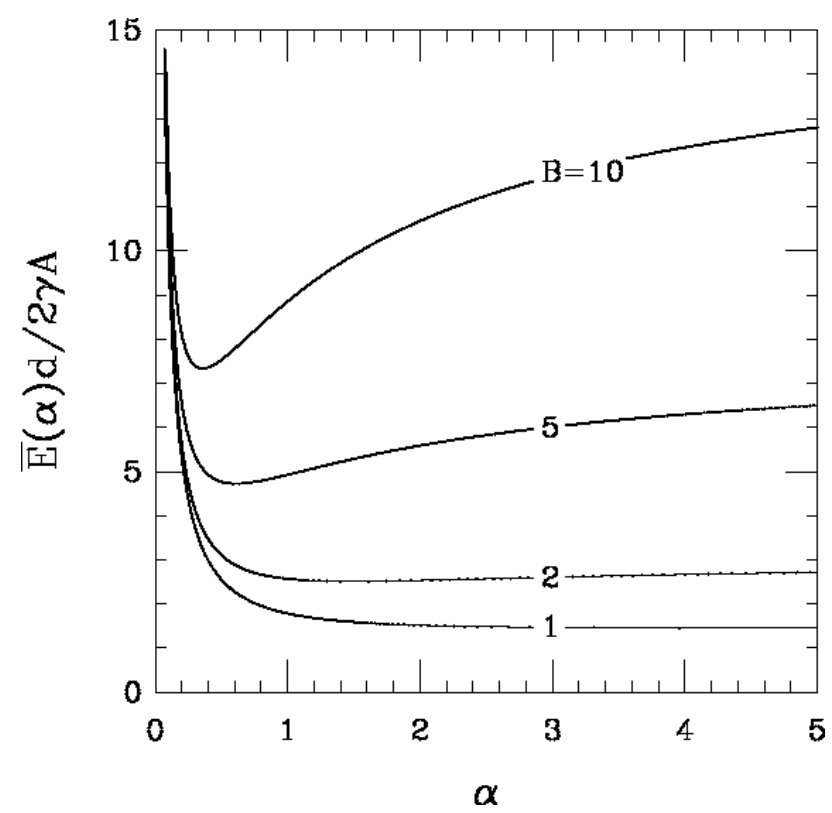

FIG. 18. Stripe energy density as a function of stripe width, for various magnetic Bond numbers.

The details of this perturbation analysis are given in Appendix A. After considerable algebra we obtain the force associated with a monochromatic perturbation of reduced wavevector $q=d k$; for the serpentine perturbation,

$$
\begin{aligned}
F_{s}(q)=q^{2}-2 N_{B} & \left\{\gamma_{E}+\ln \left(\frac{\alpha q}{2 \sqrt{1+\alpha^{2}}}\right)+K_{0}(q)\right. \\
+ & \left.K_{0}(\alpha q)-K_{0}\left(\sqrt{1+\alpha^{2}} q\right)\right\}
\end{aligned}
$$

and for the peristaltic perturbation

$$
\begin{gathered}
F_{p}(q)=q^{2}-2 N_{B}\left\{-\gamma_{E}+\ln \left(\frac{\alpha}{\sqrt{1+\alpha^{2}}}\right)-\ln (q / 2)\right. \\
\left.-K_{0}(q)-K_{0}(\alpha q)+K_{0}\left(\sqrt{1+\alpha^{2}} q\right)\right\} .
\end{gathered}
$$

Let us now look at the limit of small $q$ for serpentine perturbations,

$$
\begin{aligned}
F_{s}(q)= & \left\{1-\frac{N_{B}}{4}\left[\alpha^{2} \ln \left(1+\alpha^{-2}\right)+\ln \left(1+\alpha^{2}\right)\right]\right\} q^{2} \\
& -\frac{N_{B}}{64}\left\{\left(1+\alpha^{2}\right)^{2} \ln \left(1+\alpha^{2}\right)-\alpha^{4} \ln \left(\alpha^{2}\right)\right. \\
& \left.\quad-6 \alpha^{2}\left(1-\frac{2}{3} \gamma_{E}\right)\right\} q^{4} \\
& -\frac{N_{B}}{16} \alpha^{2} q^{4} \ln \left(\frac{1}{2} q\right)+\mathcal{O}\left(q^{6}, q^{6} \ln q\right)
\end{aligned}
$$

a)

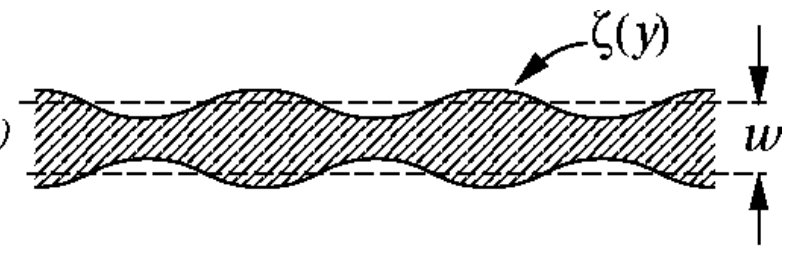

b)

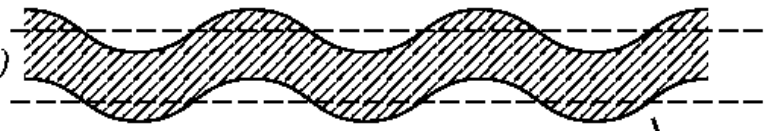

c)

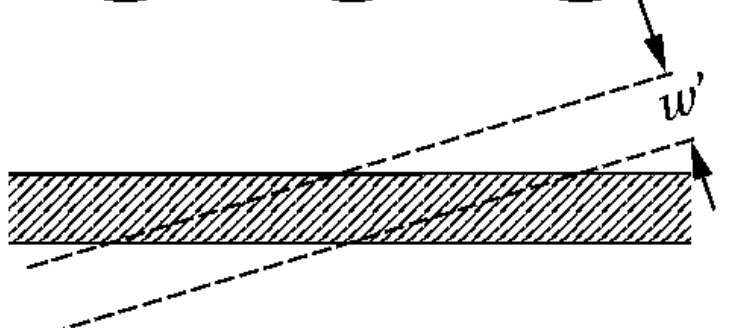

FIG. 19. Peristaltic (a) and serpentine (b) perturbations of a flux stripe. (c) Illustration of the change in stripe width upon a uniform rotation.

Serendipitously, the condition of stripe equilibrium is precisely that which sets the coefficient of $q^{2}$ to zero. This can be interpreted as a consequence of rotational invariance. Note first that by assigning the same function $\zeta(y)$ to the bottom and top edges of the stripe we have maintained the stripe width at $w$ to linear order in $\zeta$, but not at quadratic order. As shown in Fig. 19, for a uniform 
tilt of the layer boundaries, $\zeta_{y}=$ constant, the width of the rotated stripe is $w^{\prime}=w / \sqrt{1+\zeta_{y}^{2}} \simeq w-(w / 2) \zeta_{y}^{2}+\cdots$. Such a uniform tilt will cost energy through the "bulk" term $E(w)$ in (5.1) as $E\left(w^{\prime}\right)-E(w) \simeq-(w / 2) E^{\prime}(w) \zeta_{u}^{2}$, where $E^{\prime} \equiv d E / d w$. Now, the coefficient of $q^{2}$ in (5.7) has the interpretation of an effective line tension, associated with an energy

$$
E=\frac{1}{2} \gamma_{\mathrm{eff}} \int d y \zeta_{y}^{2}
$$

The rotational invariance argument thus shows that the apparent surface tension vanishes at the equilibrium stripe width. The surviving terms at $\mathcal{O}\left(q^{4}\right)$ look like bending energy of a rod,

$$
E \sim \frac{1}{2} \int d y \zeta_{y y}^{2}
$$

but this interpretation is spoiled by the term $q^{4} \ln (q)$, whose presence reflects the fundamental nonlocality of the magnetic interactions.

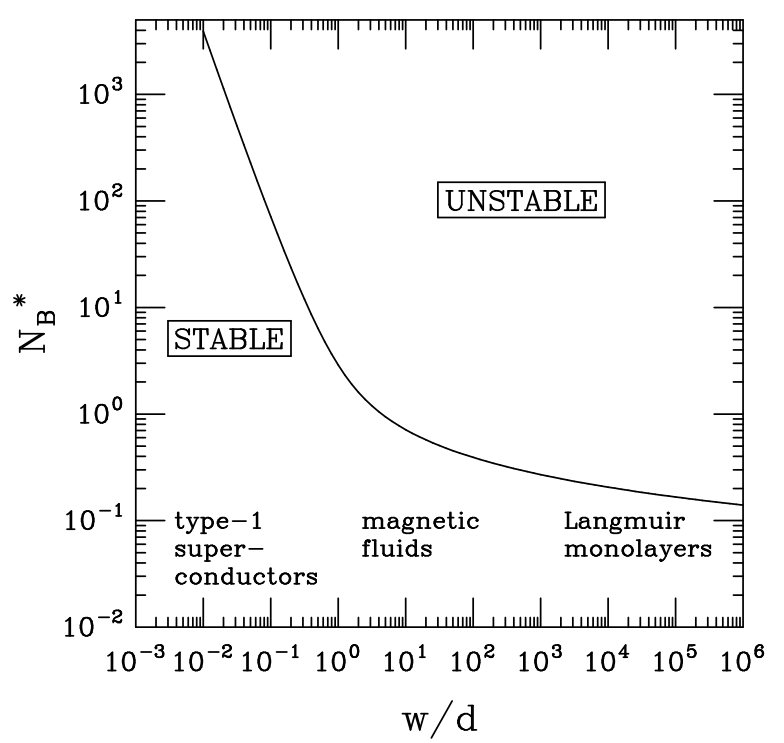

FIG. 20. Critical Bond number for serpentine instability as a function of stripe size. Typical values of $w / d$ are indicated for three experimental systems.

In the case of peristaltic perturbations, the force has a finite value as $q \rightarrow 0$, reflecting the compressibility of the stripe. The small- $q$ expansion is

$$
\begin{aligned}
F_{p}= & -2 N_{B} \ln \left(\frac{\alpha^{2}}{1+\alpha^{2}}\right)+\left\{1-\frac{N_{B}}{2}\left[\alpha^{2} \ln \alpha\right.\right. \\
& \left.\left.-\left(1+\alpha^{2}\right) \ln \sqrt{1+\alpha^{2}}\right]\right\} q^{2}+\mathcal{O}\left(q^{4}, q^{4} \ln q\right) .
\end{aligned}
$$

The equilibrium value of the stripe width as a function of Bond number, deduced in (5.3), defines the boundary of stability of stripes to serpentine perturbations. Figure 20 displays this critical Bond number $N_{B}^{*}$ as a function of $\alpha=w / d$. At fixed $\alpha$, instability occurs with increas$\operatorname{ing} N_{B}$, and likewise at fixed $N_{B}$ instability sets in with increasing $\alpha$. In the figure we have illustrated the aspect ratios $\alpha$ corresponding not only to type-I superconductors but also for typical experiments on magnetic fluids in Hele-Shaw flow (with slab thicknesses and stripe widths in the millimeter to centimeter range), and for Langmuir monolayers (with domains up to tens of microns across and a molecular thickness to the layer).

At its equilibrium width, the energy of small distortions is positive, vanishing as $q \rightarrow 0$. Thus is would seem not possible to find a field at which the stripe would be unstable to a finite-wavelength mode. This conclusion assumes that at any applied field the stripe width has its equilibrium value (5.3). Fortuitously, the elegant experimental observations on buckling instabilities in Langmuir monolayers [48] have shown us what happens when this equilibrium is not reached. Those observations concerned the dynamics of buckling when the temperature was slowly increased. Since these systems are near a critical point of phase separation, relatively small changes in temperature produce large changes in the density difference between the phases (thus altering the discontinuity in dipole density $\Delta \mu$ ) and in the line tension. These, of course, directly affect the stability of stripes, quantified by the associated electric Bond number. It was observed that slow temperature ramps produced no buckling, while rapid heating showed buckling. This suggests 49 that the dependence of stability on ramp rate is associated with a competition with mass transport as the stripe width adjusts to keep up with the temperature. Under rapid ramps, the width is out of equilibrium, yielding a nonzero (and potentially destabilizing) coefficient of $q^{2}$. Turning to the laminar state, this suggests that in the early stages of flux penetration such a mismatch between the actual and equilibrium widths allows the buckling instability to occur.

\section{B. Elastic properties of the laminar state}

By using the CL model we can also examine the elastic properties of the laminar state. This is done by considering displacements $u_{i}(y)$ of the SN interfaces away from the equilibrium laminar phase, as shown in Fig. 21. In the long wavelength (continuum) limit, $u_{i}(y)$ becomes the displacement field $u(x, y)$, and the effective elastic free energy becomes

$$
\mathcal{F}_{\mathrm{el}}=\int d^{2} r\left[\frac{B}{2}\left(u_{x}+\frac{1}{2} u_{y}^{2}\right)^{2}+\frac{K_{1}}{2} u_{y y}^{2}\right],
$$

with $B$ the bulk (compressional) modulus and $K_{1}$ the bending modulus. This result applies to serpentine per- 
turbations of the lamina; the peristaltic perturbations are gapped (like optical phonons), and do not contribute to the long wavelength properties. The general form of the free energy could have been anticipated from the single stripe calculations of the previous section; in particular, we see that the distortions in the $y$-direction appear as $u_{y y}^{2}$ (or $k_{y}^{4}|u(\mathbf{k})|^{2}$ in Fourier space), again signifying that the effective surface tension is zero. The nonlinear terms are required to preserve the rotational invariance of the free energy. The free energy, Eq. (5.11), is identical to the elastic free energy of a two dimensional smectic liquid crystal [50]. This analogy is quite useful, as the properties of two dimensional smectics have been well studied; problems such as mechanical instabilities, thermal fluctuation effects, and defect structures have been considered. We expect many of these same phenomena to occur in the laminar state.

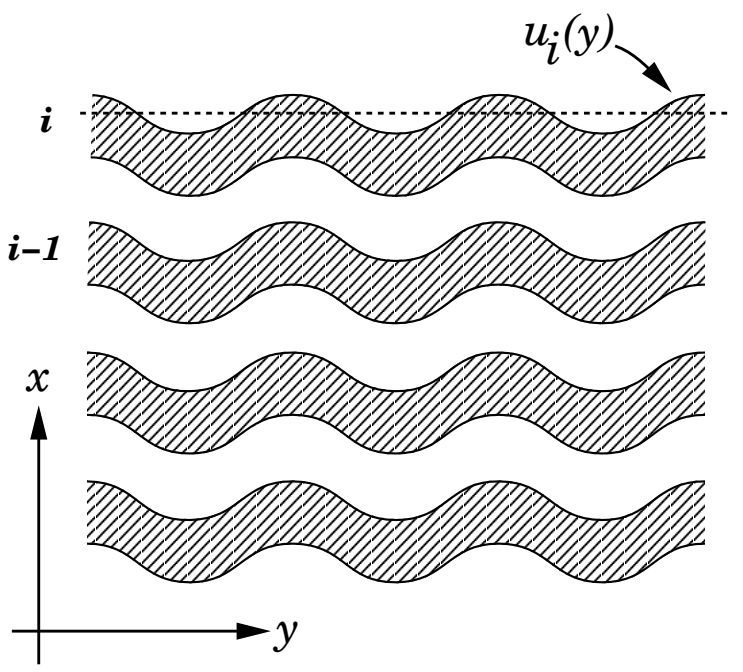

FIG. 21. Schematic diagram showing the serpentine perturbations of the domain walls. The displacement of the $i^{\text {th }}$ domain wall is $u_{i}(y)$; in the continuum limit this will become the displacement field $u(x, y)$.

The elastic moduli for striped phases in two dimensional ferromagnets with dipolar interactions have been calculated in Refs. 51,52, and for striped phases in ferrofluids in Ref. [53,54]. The calculation for the laminar phase is identical, and we include here only the final results. The bending modulus is

$$
\begin{gathered}
K_{1}=\frac{3 M^{2} a^{3}}{8 \pi^{4}} \sum_{m=1}^{\infty} \frac{\sin ^{2} m \pi h}{m^{5}}\left\{1-\left[1+\left(\frac{2 \pi d m}{a}\right)\right.\right. \\
\left.\left.+\frac{1}{3}\left(\frac{2 \pi d m}{a}\right)^{2}\right] e^{-2 \pi d m / a}\right\},
\end{gathered}
$$

where the magnetization is $M=-H_{c} / 4 \pi$, and the equilibrium spacing $a=\sqrt{\Delta d / f_{\mathrm{CL}}}$, with $f_{\mathrm{CL}}(h, d, a)$ given by Eq. (4.9). In the thick film limit this becomes

$$
K_{1}=\frac{3 M^{2} a^{3}}{8 \pi^{4}} \sum_{m=1}^{\infty} \frac{\sin ^{2} m \pi h}{m^{5}} .
$$

The bulk modulus is

$$
\begin{aligned}
B & =a^{2}\left[\frac{\partial^{2}\left(E_{C L} / A\right)}{\partial a^{2}}\right]_{h} \\
& =\frac{4 \sigma_{S N} d}{a}-\frac{8 M^{2} d^{2}}{a} \sum_{m=1}^{\infty} \frac{\sin ^{2} m \pi h}{m} e^{-2 \pi d m / a} \\
& =\frac{4 \sigma_{S N} d}{a}-\frac{2 M^{2} d^{2}}{a} \ln \left[1+\frac{\sin ^{2} \pi h}{\sinh ^{2}(\pi d / a)}\right] .
\end{aligned}
$$

In the thick film limit this becomes

$$
B=\frac{4 \sigma_{S N} d}{a} \text {. }
$$

The bending and bulk moduli may be combined to form the length scale $\tilde{\lambda}=\sqrt{K_{1} / B}$, which is a persistence length for the distortion of the laminar structure (not to be confused with the superconducting penetration depth). For thick films this length becomes

$$
\tilde{\lambda}^{2} / a^{2}=\frac{3}{32 \pi^{2}} \frac{\sum_{m=1}^{\infty} \sin ^{2}(m \pi h) / m^{5}}{\sum_{m=1}^{\infty} \sin ^{2}(m \pi h) / m^{3}} .
$$

\section{Dislocations in the laminar state}

In many of the images of the laminar state [1] one often observes edge dislocations, where half of a normal lamina has been inserted into the laminar structure (see Fig. 22 for a schematic diagram).

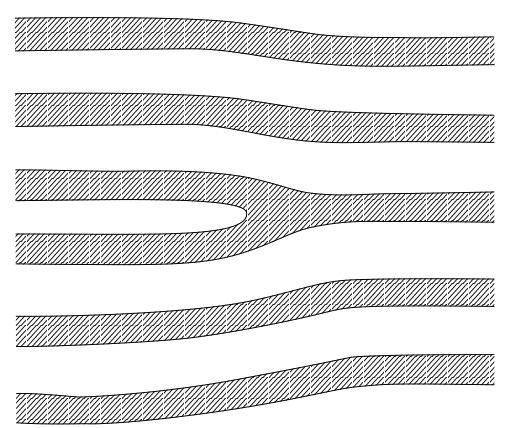

FIG. 22. A dislocation in the laminar state with a Burger's vector of 1 .

By using our elastic theory, we can determine the displacement field produced by such a dislocation; this problem has been studied in the context of 2D smectics [55] and our calculation closely follows Ref. [55]. We begin with the linearized Euler-Lagrange equations for the defect displacement field $u^{D}(x, y)$,

$$
u_{x x}^{D}-\tilde{\lambda}^{2} u_{y y y y}^{D}=\operatorname{ma} \delta^{\prime}(x) \theta(y),
$$


where a source term has been added to the right hand side to account for the presence of the dislocation, in such a way that the line integral of $\nabla u$ around the dislocation is $m a$ (the Burger's vector), with $m$ the number of half sheets inserted and $a$ the lamina spacing. Equation (5.17) may be solved using Fourier transforms [55], with the result that

$$
u^{D}(x, y)=\frac{m a}{4} \operatorname{sgn}(x)\left[\operatorname{erf}\left(\frac{y}{\sqrt{4 \tilde{\lambda}|x|}}\right)+1\right] .
$$

For an collection of dislocations centered at $\left\{\mathbf{r}_{i}\right\}$ with strengths $\left\{m_{i}\right\}$, we may introduce the dislocation density

$$
m(\mathbf{r})=\sum_{i} m_{i} \delta\left(\mathbf{r}-\mathbf{r}_{i}\right)
$$

so that the displacement field is obtained by linear superposition,

$$
u^{D}(\mathbf{r})=\int d^{2} r^{\prime} m\left(\mathbf{r}^{\prime}\right) G\left(\mathbf{r}-\mathbf{r}^{\prime}\right),
$$

with

$$
G\left(\mathbf{r}-\mathbf{r}^{\prime}\right)=\frac{a}{4} \operatorname{sgn}\left(x-x^{\prime}\right)\left[\operatorname{erf}\left(\frac{y-y^{\prime}}{\sqrt{4 \tilde{\lambda}\left|x-x^{\prime}\right|}}\right)+1\right]
$$

The effective free energy for the defects, $\mathcal{F}_{D}$, is then obtained by substituting Eq. (5.20) into the the elastic free energy, and using the "harmonic conjugate" trick of Toner and Nelson [55]. The final result is

$$
\begin{gathered}
\mathcal{F}_{D}=\frac{1}{2} \int d^{2} r_{1} \int_{\left|\mathbf{r}_{1}-\mathbf{r}_{2}\right|>a} d^{2} r_{2} m\left(\mathbf{r}_{1}\right) m\left(\mathbf{r}_{2}\right) U\left(\mathbf{r}_{1}-\mathbf{r}_{2}\right) \\
+E_{D} \int d^{2} r m^{2}(\mathbf{r})
\end{gathered}
$$

where the interaction potential is

$$
U(\mathbf{r})=\frac{a^{2} B}{4}\left(\frac{\tilde{\lambda}}{\pi|x|}\right)^{1 / 2} e^{-y^{2} / 4 \tilde{\lambda}|x|}
$$

and the defect core energy is

$$
\begin{aligned}
E_{D} & =\frac{B}{2} \int d^{2} r\left[\tilde{\lambda}^{2}\left(G_{y y}\right)^{2}+\left(G_{x}\right)^{2}\right] \\
& =\frac{1}{8 \sqrt{\pi}} B a^{2}\left(\frac{\tilde{\lambda}}{a}\right)^{1 / 2} .
\end{aligned}
$$

The core energy can be calculated as a function of the reduced field $h$ by using the results of the previous section [Eqs. (5.14) and (5.16)]. A rough estimate shows that this energy is generally of order $10^{-3}\left(H_{c}^{2} / 8 \pi\right) a^{3}$, and can therefore be quite small; as a result it should be easy to nucleate dislocations in the laminar phase.

\section{DISCUSSION}

The free-boundary approach to the energetics and dynamics of the intermediate state has led us to a clearer understanding of the shape instabilities of individual flux domains and ordered arrays. At the same time, the correspondence with smectic liquid crystals suggests that phenomena observed there should have an analog in type-I superconductors. Below we suggest several experiments to visualize these phenomena. These have as their starting point the ordered laminar state, produced with an inplane component to the field. Preliminary experiments by Reisin and Lipson [56] have shown some of these phenomena.

(i) The buckling instability: Rapid changes in the magnitude of the applied normal field may allow buckling instabilities to occur in much the same way as observed in Langmuir monolayers. Of interest would be the dependence of buckling wavelength on the magnitude of the field jump.

(ii) The chevron instability: If the in-plane magnetic field is applied at an angle with respect to the lamina we expect an instability toward a zig-zag or chevron pattern as the lamina attempt to reorient. This is the analog of the Helfrich-Hurault effect in smectics [50], wherein a field component normal to smectic layers, producing a torque on them, induces an undulatory instability.

(iii) The Eckhaus instability: If the magnetic field normal to the slab is slowly increased or decreased in magnitude the stripe width and spacing must adjust to stay in equilibrium. As in convective systems [57, this may occur through an Eckhaus-like nucleation phenomenon to create or destroy laminae. Dislocations can be produced which will move toward the sample edges or annihilate at the center in accord with the direction in which the wavelength must adjust. Their climb and glide dynamics will provide an important testing ground for the theory of superconductor-normal interface dynamics.

(iv) Critical-point effects: In the simplified contour dynamics in which the local field is taken to be the critical field we saw that the effective Bond number depended only on the ratio $d / \Delta(T)$. Near the zero-field critical temperature for the superconductor-metal transition the interfacial width $\Delta(T)$ diverges with reduced temperature $\left(T_{c}-T\right) / T_{c}$. This should produce characteristic changes in the equilibrium stripe width as well as possibly inducing shape transformations.

We close by emphasizing what has not been accomplished in this study. First, we have considered laterally infinite samples, so that the whole issue of flux penetration at the edges is ignored. This is known to be very significant in both type-I and type-II superconductors [58]. A treatment of these effects requires not only the electromagnetics of the fields in the neighborhood of the slab edges but also consideration of processes such as domain 
fission. Second, we have presented a highly oversimplified dynamical picture in which diffusional instabilities are absent and only mechanical ones appear. The interplay between the Mullins-Sekerka and these mechanical instabilities appears not to have been considered theoretically and may shed some light on various problems in flux domain pattern formation. Third, a large-scale numerical study of the many-domain problem has not been attempted, precluding a clear understanding of the true "energy landscape" of this strongly interacting system. Fourth, the effects of in-plane components to the applied magnetic fields has not been incorporated into the freeboundary approach in any quantitative way. This will be important for a quantitative understanding of the instabilities described above. Fifth, the extension of matched asymptotic methods used in purely two-dimensional systems to the slab geometry in which the intermediate state appears has not been developed. A detailed study of this point would greatly clarify the free-boundary approach to flux domain shapes. Finally, coarse-graining approaches to domain dynamics analogous to Otto and Kohn's recent study of magnetic fluid pattern formation may prove quite fruitful [59,60].

\section{ACKNOWLEDGMENTS}

We are grateful to C.-Y. Mou, Thomas R. Powers, and John Toner for important discussions on the energetics of the intermediate state, to O. Narayan and H. Bokil for communication of Ref. [18] prior to publication, and to C. Reisin and S.G. Lipson for sharing with us the results of their ongoing experiments. This work was supported in part by NSF PFF Grant No. DMR 93-50227 (REG), No. DMR 92-23586 and 96-28926 (ATD), and the Alfred P. Sloan Foundation (REG and ATD).

\section{APPENDIX A: FLUX-STRIPE STABILITY CALCULATION}

Here we collect some details of the stability analysis for single flux stripes. In the case of peristaltic distortions,

$$
\mathbf{r}_{ \pm}(y)=y \hat{\mathbf{e}}_{y}+\left( \pm \frac{w}{2} \pm \zeta(y)\right) \hat{\mathbf{e}}_{x}
$$

The linearized normal force can be reduced to the form

$$
\begin{aligned}
U(y)= & \gamma \zeta_{y y} \\
& +2 M^{2} \int_{-\infty}^{\infty} d y^{\prime}\left[\zeta\left(y^{\prime}\right)+\zeta(y)\right]\left[S_{d^{2}+w^{2}}-S_{w^{2}}\right] \\
& +2 M^{2} \int_{-\infty}^{\infty} d y^{\prime}\left[\zeta\left(y^{\prime}\right)-\zeta(y)\right]\left[S_{d^{2}}-S_{0}\right],
\end{aligned}
$$

where $S_{a^{2}}=1 /\left|\left(y^{\prime}-y\right)^{2}+a^{2}\right|$. For serpentine distortions, the displacements are

$$
\mathbf{r}_{ \pm}(y)=y \hat{\mathbf{e}}_{y}+\left( \pm \frac{w}{2}+\zeta(y)\right) \hat{\mathbf{e}}_{x},
$$

and the normal force has the simpler form

$$
\begin{aligned}
U(y)= & \gamma \zeta_{y y} \\
& +2 M^{2} \int_{-\infty}^{\infty} d y^{\prime}\left[\zeta\left(y^{\prime}\right)-\zeta(y)\right] \\
& \times\left[S_{d^{2}+w^{2}}-S_{w^{2}}-S_{d^{2}}-S_{0}\right]
\end{aligned}
$$

If the distortion is the plane wave $\zeta(y)=A \cos (k y)$, then the force is proportional to $\zeta$ with a coefficient $F(k)$

$$
\begin{aligned}
F(k)=-\gamma k^{2}+4 M^{2} \int_{0}^{\infty} d y[1-\cos (k y)] \\
\quad \times\left[S_{d^{2}+w^{2}}-S_{w^{2}}-S_{d^{2}}+S_{0}\right]
\end{aligned}
$$

where now $S_{a^{2}}=1 /\left|y^{2}+a^{2}\right|$. Several of the integrals with integrands proportional to $\cos (k y)$ reduce trivially to Bessel functions, but care must be taken to account for canceling divergences in the remaining terms. This is conveniently done by considering the limiting process

$$
I=\lim _{u \rightarrow \infty} \int_{0}^{u} d y\left\{S_{d^{2}+w^{2}}-S_{w^{2}}-S_{d^{2}}+\frac{1-\cos (k y)}{y}\right\} .
$$

Rescaling these equations, we obtain

$$
\begin{aligned}
I=\lim _{u \rightarrow \infty} & \left\{\int_{0}^{u / \sqrt{w^{2}+h^{2}}} d y S_{1}-\int_{0}^{u / w} d y S_{1}\right. \\
& \left.-\int_{0}^{u / h} d y S_{1}+\int_{0}^{u k} d y \frac{1-\cos (y)}{y}\right\}
\end{aligned}
$$

A useful intermediate result at this stage is 61

$$
\int_{0}^{u} d y \frac{1-\cos (y)}{y}=\gamma_{E}+\ln (u)+\int_{u}^{\infty} d y \frac{\cos (y)}{y},
$$

where $\gamma_{E}=0.577215 \ldots$ is Euler's constant. Substitution into A7 and evaluation of the remaining integrals then yields the final results $(5.5)$ and $(5.6)$ for the energy of serpentine and peristaltic perturbations.

* $\quad$ Electronic mail: dorsey@phys.ufl.edu

** Electronic mail: gold@physics.arizona.edu.

[1] R.P. Huebener, Magnetic Flux Structures in Superconductors (Springer-Verlag, New York, 1979).

[2] T.E. Faber, Proc. Roy. Soc. A 248, 460 (1958).

[3] F. Haenssler and L. Rinderer, Helv. Phys. Acta 40, 659 (1967). 
[4] R.N. Goren and M. Tinkham, J. Low Temp. Phys. 5, 465 (1971).

[5] R.P. Huebener, R.T. Kampwirth, and V.A. Rowe, Cryogenics 12, 100 (1972).

[6] I.V. Sharvin, Sov. Phys. JETP 6, 1031 (1958).

[7] L.D. Landau, Sov. Phys. JETP 7, 371 (1937).

[8] E.R. Andrew, Proc. Roy. Soc. (London) A194, 98 (1948); K. Maki, Ann. Phys. 34, 363 (1965); G. Lasher, Phys. Rev. 154, 345 (1967); D.J.E. Callaway, Ann. Phys. 213, 166 (1992).

[9] H. Frahm, S. Ullah, and A.T. Dorsey, Phys. Rev. Lett. 66, 3067 (1991).

[10] F. Liu, M. Mondello, and N. Goldenfeld, Phys. Rev. Lett. 66, 3071 (1991).

[11] A.B. Pippard, Phil. Mag. 41, 243 (1950).

[12] W.W. Mullins and R.F. Sekerka, J. Appl. Phys. 35, 444 (1964).

[13] A.T. Dorsey, Ann. Phys. 233, 248 (1994); J.C. Osborn and A.T. Dorsey, Phys. Rev. B 50, 15961 (1994); S. J. Chapman, Quart. Appl. Math. 53, 601 (1995).

[14] J. Pearl, Appl. Phys. Lett. 5, 65 (1964).

[15] A.L. Fetter and P.C. Hohenberg, Phys. Rev. 159, 330 (1967).

[16] R.E. Goldstein, D.P. Jackson, and A.T. Dorsey, Phys. Rev. Lett. 76, 3818 (1996).

[17] For a review, see D.A. Kessler, J. Koplik, and H. Levine, Adv. Phys. 37, 255 (1988).

[18] See H. Bokil and O. Narayan, "Flux penetration in slab shaped Type-I superconductors," cond-mat/9610039.

[19] M. Seul and D. Andelman, Science 267, 476 (1995).

[20] R.E. Rosensweig, M. Zahn, and R. Shumovich, J. Magn. Magn. Mater. 39, 127 (1983).

[21] A.O. Tsebers and M.M. Mairov, Magnetohydrodynamics 16, 21 (1980).

[22] A.J. Dickstein, S. Erramilli, R.E. Goldstein, D.P. Jackson, and S.A. Langer, Science 261, 1012 (1993).

[23] S.A. Langer, R.E. Goldstein, and D.P. Jackson, Phys. Rev. A 46, 4894 (1992).

[24] D.P. Jackson, R.E. Goldstein, and A.O. Cebers, Phys. Rev. E 50, 298 (1994).

[25] D. Andelman, F. Brochard, and J.-F. Joanny, J. Chem. Phys. 86, 3673 (1987).

[26] K.Y.C. Lee and H.M. McConnell, J. Phys. Chem. 97, 9532 (1993).

[27] R.E. Goldstein and D.P. Jackson, J. Phys. Chem. 98, 9626 (1994).

[28] M. Seul, L.R. Monar, L.O'Gorman, and R. Wolfe, Science 254, 1616 (1991).

[29] D.M. Petrich and R.E. Goldstein, Phys. Rev. Lett. 72, 1120 (1994).

[30] R.E. Goldstein, D.J. Muraki, and D.M. Petrich, Phys. Rev. E 53, 3933 (1996).

[31] A. Hagberg and E. Meron, Phys. Rev. Lett. 72, 2494 (1994).

[32] C.B. Muratov and V.V. Osipov, Phys. Rev. E 53, 3101 (1996).

[33] K.J. Lee, W.D. McCormick, Q. Ouyang, and H.L. Swinney, Science 261, 192 (1993).

[34] K.J. Lee and H.L. Swinney, Phys. Rev. E 51, 1899 (1995).

[35] R. FitzHugh, Biophys. J. 1, 445 (1961); J.S. Nagumo, S. Arimoto, and Y. Yoshizawa, Proc. IRE 50, 2061 (1962);
R. FitzHugh, in Biological Engineering, ed. H.P. Schwan (McGraw-Hill, New York, 1969).

[36] L. M. Milne-Thomson, Theoretical Hydrodynamics, Fifth Edition (MacMillan Press Ltd., London 1968), Chapters $\mathrm{XI}$ and XII.

[37] G. Birkhoff and E. H. Zarantonello, Jets, Wakes, and Cavities (Academic Press, New York 1957).

[38] L.D. Landau, E.M. Lifshitz, and L.P. Pitaevskii, Electrodynamics of Continuous Media, 2nd ed. (Pergamon Press, New York 1984), p. 119.

[39] A. Fortini and E. Paumier, Phys. Rev. B 5, 1850 (1972).

[40] The energy of a sample of volume $V$ with a uniform magnetization $\mathbf{M}$ in an external field $\mathbf{H}_{a}$ is $-\frac{1}{2} \mathbf{M} \cdot \mathbf{H}_{a} V$. For a superconductor, $\mathbf{M}=-\mathbf{H}_{n} / 4 \pi$, so that the energy per unit area of the sample is $H_{n} H_{a} d / 4 \pi$.

[41] This ignores the added area of the SN interface due to the flaring of the normal domains.

[42] C.-Y. Mou, private communication.

[43] C. Kooy and V. Enz, Philips Res. Rep. 15, 7 (1960).

[44] J. A. Cape and G. W. Lehman, J. App. Phys. 42, 5732 (1971).

[45] See A.A. Abrikosov, Fundamentals of the Theory of Metals (North-Holland, Amsterdam 1988), p. 479.

[46] For a discussion of surface tension anisotropy within the context of dendritic growth, see Ref. 17].

[47] A. Bodmer, U. Essman, H. Träuble, Phys. Stat. Solidi (a) 13, 471 (1972); A. Bodmer, ibid. 19, 513 (1973).

[48] K.J. Stine, C.M. Knobler, and R.C. Desai, Phys. Rev. Lett. 65, 1004 (1990).

[49] A.O. Cebers and R.E. Goldstein, unpublished.

[50] W. Helfrich, Appl. Phys. Lett. 17, 531 (1970); J.P. Hurault, J. Chem. Phys. 59, 2086 (1973); See also P.G. de Gennes and J. Prost, The Physics of Liquid Crystals (Oxford University Press, New York 1993), pp. 361-4.

[51] A. Kashuba and V.L. Pokrovsky, Phys. Rev. Lett. 70, 3155 (1993); Phys. Rev. B 48, 10355 (1993). Some errors in the calculation of the bending modulus are corrected in Appendix A of Ar. Abanov, V. Kalatsky, V.L. Pokrovsky, and W.M. Saslow, Phys. Rev. B 51, 1023 (1995).

[52] K.-O. Ng and D. Vanderbilt, Phys. Rev. B 52, 2177 (1995).

[53] A. Cebers, J. Magn. Mag. Mat. 149, 93 (1995).

[54] C. Flament, J.C. Bacri, A. Cebers, F. Elias, R. Perzynski, Europhys. Lett. 34, 225 (1996).

[55] J. Toner and D. R. Nelson, Phys. Rev. B 23, 316 (1981).

[56] C.R. Reisin and S.G. Lipson, "Period Dependence of the Intermediate State Structures of Type-I Superconductors," preprint (1997), and private communication.

[57] M.C. Cross and P.C. Hohenberg, Rev. Mod. Phys. 65, 851 (1993).

[58] C.A. Duran, P.L. Gammel, R.E. Miller, and D.J. Bishop, Phys. Rev. B 52, 75 (1995).

[59] F. Otto, "Dynamics of labyrinthine pattern formation in magnetic fluids: a mean field theory," Arch. Rat. Mech. Analysis, in press (1997).

[60] F. Otto and R. Kohn, "Small surface energy, coarsegraining, and selection of microstructure," Physica D (in press).

[61] I.S. Gradshteyn and I.M. Ryzhik, Table of Integrals, Series, and Products, Fifth Edition (Academic Press, Inc., San Diego 1994), \# 3.782.1. 
TABLE I. Analogies between free streamline flow in fluids and lamina formation in the intermediate state of type-I superconductors.

\begin{tabular}{ll}
\hline \hline Free streamline flow around a plate & Laminae in superconductors \\
\hline Complex potential $w=\phi+i \psi$ & Complex potential $w=\phi+i A_{y}$ \\
Complex fluid velocity $u-i v=-d w / d \zeta$ & Complex magnetic field $B=B_{x}-i B_{z}=-d w / d \zeta$ \\
Streamlines & Field lines (lines of force) \\
Free streamline & Superconducting-normal interface \\
Free streamline velocity $U$ & Superconducting critical field $H_{c}$ \\
Region of fluid flow & Normal phase with nonzero magnetic field \\
Cavity behind plate & Superconducting phase \\
Riabouchinsky flow & Lamina in a finite thickness plate \\
\hline \hline
\end{tabular}


TABLE II. Analogies between interfacial energetics of type-I superconductors and other systems. The energy of a set $\left\{\mathcal{D}_{i}\right\}$ of domains is written as $E\left[\left\{\mathbf{r}_{i}\right\}\right]=\Pi \sum_{i} A_{i}+\gamma \sum_{i} L_{i}-\frac{1}{2} \Omega \oint d s \oint d s^{\prime} \hat{\mathbf{t}}_{i} \cdot \hat{\mathbf{t}}_{j} \Phi_{i j}\left(R_{i j} / \xi\right)$.

\begin{tabular}{|c|c|c|c|c|c|}
\hline System & $\Pi$ & $\gamma$ & $\Omega$ & $\Phi$ & $\xi$ \\
\hline type-I superconductors ${ }^{a}$ & $\left(H_{c}^{2} d / 8 \pi\right)\left(\rho_{n}+h^{2} / \rho_{n}\right)$ & $H_{c}^{2} d \Delta / 8 \pi$ & $H_{a}^{2} d / 8 \pi^{2}$ & $\sinh ^{-1}(1 / z)+z-\sqrt{1+z^{2}}$ & $d$ \\
\hline magnetic fluids ${ }^{b}$ & Lagrange multiplier & $d \sigma_{F W}$ & $2 d M^{2}$ & $\sinh ^{-1}(1 / z)+z-\sqrt{1+z^{2}}$ & $d$ \\
\hline Langmuir monolayers $^{c}$ & Lagrange multiplier & $\gamma_{L E-L C}$ & $(\Delta \mu)^{2}$ & $1 / 2 z^{*}$ & $d_{\mathrm{mol}}$ \\
\hline
\end{tabular}

Explanation of symbols: $\sigma_{F W}$, ferrofluid water surface tension; $M$, ferrofluid magnetization; $\gamma_{L E-L C}$, line tension between liquid expanded (LE) and liquid condensed (LC) phases in a Langmuir monolayer; $\Delta \mu$, discontinuity in electric dipole moment density between LE and LC phases; $d_{\text {mol }}$, a molecular cutoff - monolayer thickness.

a Present work.

$b$ Refs. 23.24.

$c$ Refs. 26, 27.

${ }^{d}$ Eq. (2.9) and Refs. 29,30.

* This limiting form is supplemented with a cutoff procedure. See Ref. [27].

** The system of units in Eq. (2.9) sets the inhibitor screening length to unity. 